\title{
Overlapping Unstructured Grid Technology for the Numerical Simulation of Flow Around Multistage Space System
}

\author{
A.L. Zheleznyakova \\ A.Yu. Ishlinsky Institute for Problems in Mechanics Russian Academy of Sciences, \\ 119526, Moscow, Russia \\ a.zheleznyakova@mail.ru
}

\begin{abstract}
The application of the composite grid concept in the framework of unstructured approach for the numerical simulation of flow around complex multistage space systems is discussed. The group of streamlined bodies of arbitrary configuration under the changes in the number of system elements and their mutual arrangement is considered. This technology is based on the application of overlapping unstructured grids, which are independently generated for each object of the system. The proposed aerodynamic model of system of arbitrarily located objects having complex configurations is based on the computational technologies for unstructured mesh generation and for the integration of the Navier - Stokes / Euler equations, which are developed in Institute for Problems in Mechanics Russian Academy of Sciences (IPMech RAS). The algorithm implementation requires minor source code modifications. The possibilities of the approach are demonstrated by the example of the numerical simulation of flow around complex multistage space transportation system Space Shuttle. The created virtual prototype of the space system arrangement unites in itself the set of volume grids, which are independently generated for individual stages based on the most realistic virtual surfaces. A mathematical description of the geometry of each system element contains all the basic details of the corresponding configuration.
\end{abstract}

Keywords: composite computational mesh, overlapping grids, unstructured mesh, group of streamlined bodies, aerodynamic arrangement, reusable space transportation system, gas dynamics, numerical simulation, computational aerodynamics, software systems, computer-aided design (CAD), virtual prototype, comprehensive 3D models.

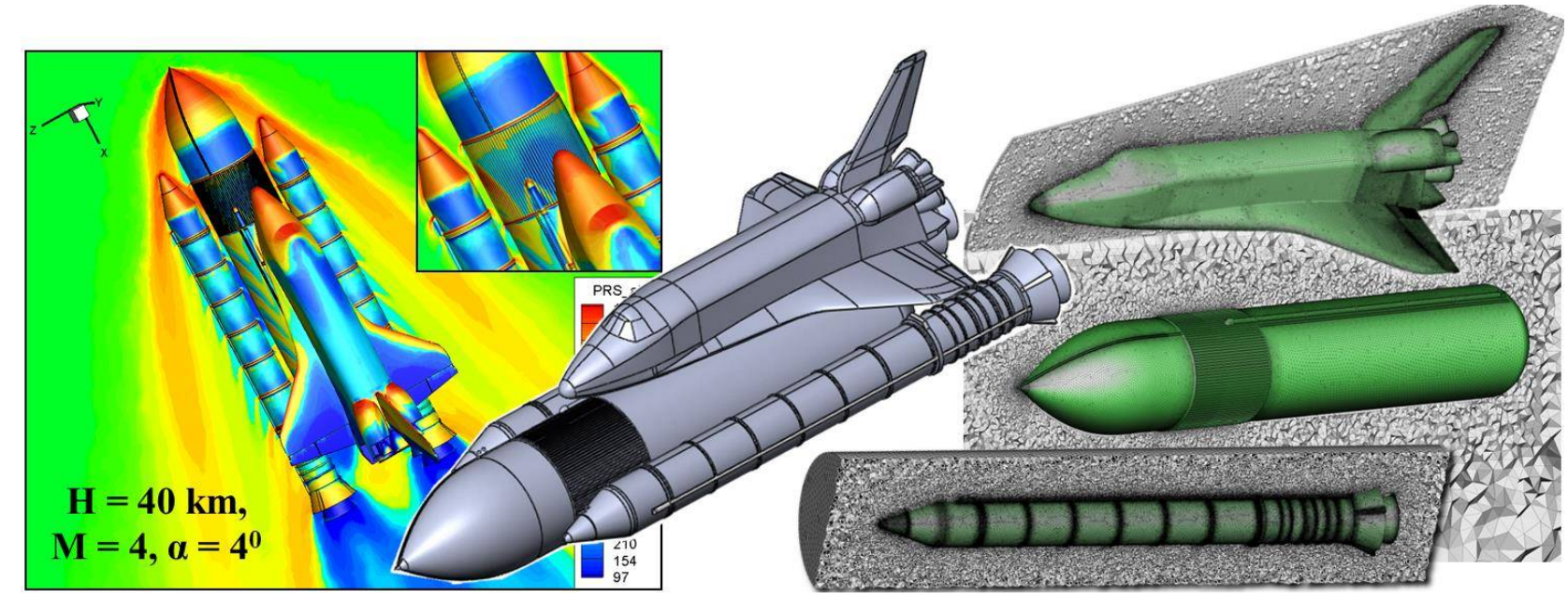

The computer Space Shuttle system geometry, independently generated components of composite grid and the flow structure (pressure field, $\mathrm{Pa}$, distribution) around a complex configuration under the conditions corresponding to the point along an ascent trajectory 


\title{
Технология композитных неструктурированных сеток для компьютерного моделирования обтекания многоступенчатой космической системы
}

\author{
А.Л. Железнякова \\ Институт проблем механики им. А.Ю. Иилинского Российской академии наук, \\ 119526, Москва, проспект Вернадского, 101-1 \\ a.zheleznyakova@mail.ru
}

\begin{abstract}
Аннотация
Обсуждается применение концепции композитных сеток в рамках неструктурированного подхода для численного моделирования обтекания сложных многоступенчатых аэрокосмических систем. Рассматривается группа совместно обтекаемых тел произвольной конфигурации с изменяемым взаимным положением и числом объектов, входящих в систему. Технология основана на использовании неструктурированных перекрывающихся пространственных сеток, независимо сгенерированных для каждого объекта исследуемой системы. Предложенная численная модель аэродинамики системы произвольно расположенных тел сложной конфигурации построена на основе вычислительных технологий создания неструктурированных сеточных моделей и решения полной системы уравнений механики сплошной среды (уравнений Навье - Стокса), разработанных в ИПМех РАН. Программная реализация расчетной модели не требует значительной модификации исходных кодов. Возможности подхода продемонстрированы на примере численного моделирования обтекания космической транспортной системы Space Shuttle во взлетной конфигурации, включающей орбитальный космоплан, внешний топливный бак и твердотопливные ускорители. Созданный виртуальный прототип полной компоновки многоразовой космической системы объединяет в себе набор объемных сеточных моделей отдельных ступеней, независимо сгенерированных на основе максимально реалистичных компьютерных поверхностей. Математическое описание геометрии каждого модуля содержит все основные детали и конструктивные элементы соответствующего прообраза.

Ключевые слова: композитная расчетная сетка, сетки с перекрытием, неструктурированные сетки, обтекание группы тел, аэродинамическая компоновка, многоразовая космическая транспортная система, газовая динамика, математическое моделирование, вычислительная аэродинамика, программные комплексы, автоматизированное проектирование, виртуальный прототип, трехмерные реалистичные модели.
\end{abstract}

\section{1. Введение}

За всю историю освоения космического пространства было реализовано лишь две концепции многоразовой транспортной космической системы - МТКС Space Shuttle [1-3] и “Энергия - Буран” [4]. При этом корабль “Буран” выполнил единственную космическую миссию в беспилотном режиме, а регулярные запуски системы Space Shuttle, осуществлявшиеся с 1981 по 2011 гг., выявили нерентабельность и экономическую неэффективность эксплуатации последней. Изначально проект "Космическая транспортная система" (Space Transportation System, STS), предполагал многоразовое использование всех элементов системы: твердотопливных ускорителей, внешнего топливного бака, орбитального ракетоплана 
(рис.1). За счет многократного применения дорогостоящего оборудования и челночной доставки грузов планировалось существенно снизить стоимость вывода полезной нагрузки на низкую околоземную орбиту. На практике многоразовому использованию подвергался только орбитальный модуль. Большой подвесной топливный отсек отделялся через 8.5 минут после старта на высоте 113 км и сгорал в атмосфере. Хотя спасение отработанных боковых ускорителей с помощью парашютов было предусмотрено, на их ремонт и перезаправку затрачивалось немногим меньше средств, чем на строительство новых [1-3].

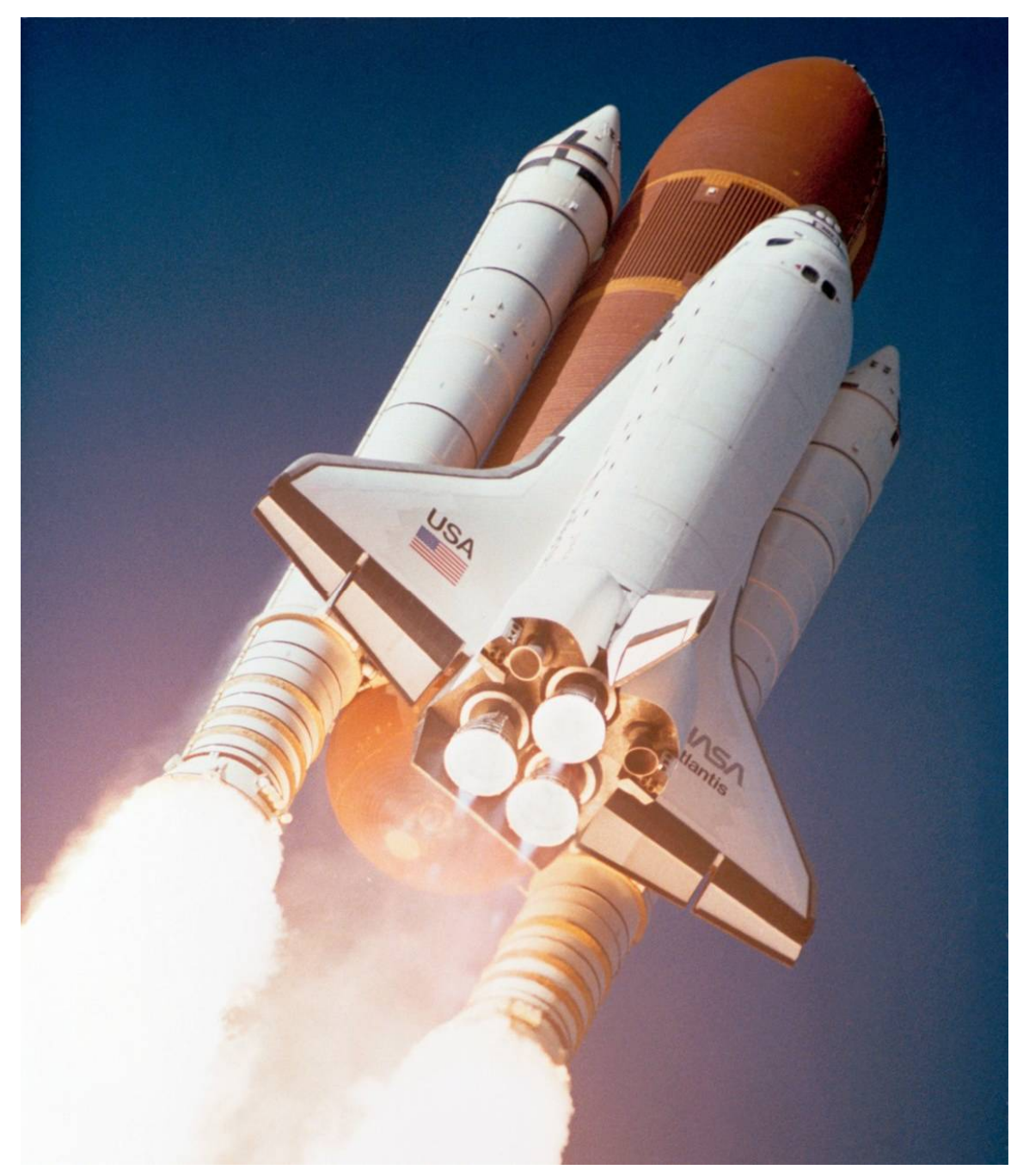

Рис.1. Взлетная конфигурация многоразовой транспортной космической системы Space Shuttle [5]

В течение 30 лет эксплуатации пять орбитальных самолетов (Columbia, Challenger, Discovery, Atlantis и Endeavour) осуществили 135 полетов в космос (от 10 до 39 полетов каждый), хотя изначально планировалось проводить по 24 старта в год. Кроме того, предполагалось, что корабль серии Space Shuttle сможет совершить до 100 миссий. Проектные энергомассовые характеристики комической транспортной системы также не были достигнуты. Транспортный корабль, рассчитанный на челночную доставку крупных грузов, в действительности смог обеспечить вывод полезной нагрузки не более 24.4 т на низкую опорную круговую орбиту высотой 200 км с минимальным наклонением около $28^{\circ}$ (широта космодрома Канаверал) и возвращение грузов до 14.5 т. Более того, стоимость доставки 1 кг полезного груза на орбиту почти в 2 раза превысила аналогичный показатель для одноразовой ракетыносителя [1-3]. 
Несмотря на то, что многоразовые космические системы Space Shuttle совершали максимум 8 стартов в год, имели очень ограниченный круг применений (использовались в основном для исследовательских целей и обслуживания орбитальных аппаратов) и не оправдали в полной мере возлагавшихся на них надежд, концепция МТКС не теряет своей актуальности. Пятьдесят лет назад идея использования возвращаемых ступеней была преждевременной, однако в настоящее время стал возможен дальнейший прогресс в области разработки космических транспортных систем нового поколения благодаря развитию современных технологий и появлению инновационных материалов. Успех данного направления определяется комплексным подходом к решению практических задач аэротермодинамики сложных многоступенчатых космических систем, который объединяет экспериментальные и расчетнотеоретические методики. Стремительный прогресс наблюдается в области компьютерных технологий проектирования [6-10] вследствие непрерывного наращивания производительности и быстродействия процессоров, увеличения емкости запоминающих устройств, создания удобных пользовательских интерфейсов. Быстрому развитию данного направления также способствует совершенствование вычислительных методов, возрастающая надежность компьютерных моделей, наряду со снижением вычислительных затрат.

Современные технологии автоматизированного проектирования и методы численного анализа эффективно применяются на всех этапах создания перспективных аэрокосмических изделий от разработки концепции и формирования технического облика до компьютерного сопровождения наземных и летных испытаний прототипов. Математическое моделирование используется для экстраполяции результатов наземных экспериментов на реальные условия полета, численного экспресс-анализа для выбора оптимальной траектории, быстрой интерпретации экспериментальных данных. Использование вычислительных технологий при создании аэродинамической базы изделия позволяет существенно сократить финансовые затраты и сроки работ по оптимизации характеристик летательного аппарата, уменьшить объем дорогостоящих стендовых и летных испытаний. Кроме этого, в рамках численного подхода возможен расчет параметров, недоступных для экспериментального определения. Расчетные методики позволяют находить практически непрерывные распределения тепловых и динамических характеристик поверхности летательного аппарата, в то время как в ходе испытаний (особенно в условиях летного эксперимента) значения соответствующих величин удается получить лишь в точках расположения измерительной аппаратуры.

Для компьютерной поддержки проектирования сложных многоступенчатых космических систем необходимо создавать специализированные программные средства, которые обеспечивают возможность рассмотрения большого числа режимов и взаимных положений объектов в кратчайшие сроки при приемлемой точности численных результатов, а также учитывают особенности течения. В частности, реалистичное имитационное моделирование системы пространственных объектов произвольной конфигурации требует учета сложных явлений аэродинамической интерференции на всех стадиях, включающих совместное движение ступеней, этап их разделения и полет одного объекта вблизи поверхности другого. Вследствие формирования сложной структуры течения около близко расположенных тел, на их поверхностях образуются различные по протяженности области повышенного и пониженного давления, что приводит к изменению локальных и интегральных тепловых и аэродинамических характеристик исследуемых конфигураций. Очевидно, что определение величин аэродинамических и тепловых нагрузок не может вестись без изучения сложного пространственного течения и точного определения газодинамических параметров в окрестности обтекаемых объектов. Учитывая, что экспериментальное исследование обтекания многоступенчатых космических кораблей в полной компоновке трудноосуществимо и дорого, значение математического моделирования в решении проблем проектирования таких систем сложно переоценить. 
В настоящей работе обсуждается универсальный подход к моделированию обтекания реалистичных моделей многоступенчатых космических систем в полной компоновке при изменении числа и взаимного расположения ступеней (в процессе их разделения). Предложенная технология основана на использовании композитной неструктурированной сеточной модели, которая объединяет в себе набор из двух или более перекрывающихся сеток, независимо сгенерированных около каждого объекта системы. Демонстрируется, что рассматриваемая методика успешно применяется не только в рамках блочно-структурированного подхода. Перекрывающиеся неструктурированные сетки могут также эффективно использоваться для моделирования сложных течений, благодаря возможности дискретизировать расчетную область произвольной формы и относительно легко адаптироваться в зонах больших градиентов. Кроме этого, в отличие от альтернативной блочно-структурированной технологии, число сеток не возрастает с увеличением сложности геометрии расчетной области. Последнее преимущество позволяет сократить вычислительные затраты при интерполяции данных между сетками. При использовании подхода, основанного на независимом создании расчетных сеток около каждого модуля многоступенчатой космической системы, удается избежать перестроения сложных сеточных моделей при изменении взаимного положения элементов системы. Учитывая, что трудоемкость процесса создания расчетной сетки для реалистичных моделей изделий авиационной и ракетно-космической техники составляет около двух третей от общего времени моделирования, применение предложенной методики может привести к значительной экономии вычислительных ресурсов.

Рассматриваемая численная модель аэродинамики системы произвольно расположенных тел сложной конфигурации построена на основе вычислительных технологий создания неструктурированных сеточных моделей [11-14] и решения полной системы уравнений сплошной среды (уравнений Навье - Стокса), разработанных в Институте проблем механики им. А.Ю. Ишлинского РАН (ИПМех РАН) [15-23]. Программная реализация композитной модели не потребовала значительной модификации исходных кодов. При относительно небольших дополнительных затратах вычислительных ресурсов на интерполяцию данных с сетки на сетку, подход дает значительный выигрыш в производительности, так как нет необходимости перестраивать сетки при изменении взаимного положения элементов сложной системы.

Возможности разработанной технологии демонстрируются на примере построения композитной сетки для численного моделирования обтекания космической транспортной системы Space Shuttle во взлетной конфигурации, включающей орбитальный космоплан, внешний топливный бак и твердотопливные ускорители (рис.1). Созданный виртуальный прототип полной компоновки многоразовой космической системы объединяет в себе набор объемных сеточных моделей, независимо сгенерированных для каждой ступени на основе максимально реалистичных компьютерных поверхностей.

\section{2. Виртуальные геометрические модели отдельных модулей космической транспортной системы Space Shuttle}

В качестве прообраза виртуального прототипа сложной космической системы была выбрана конфигурация двухступенчатого многоразового транспортного космического корабля Space Shuttle Atlantis (рис.1). Главным критерием отбора являлось наличие большого объема общедоступных информационных ресурсов, включающих чертежи и конструктивно-компоновочные схемы всей системы и отдельных модулей, по которым удалось воссоздать достаточно реалистичную виртуальную модель поверхности космического корабля [1-3, 24-30]. Математическое описание геометрии каждой ступени Space Shuttle содержит все основные детали и конструктивные элементы соответствующего прообраза, однако построенные компьютерные модели не претендуют на полное соответствие реальному изделию. Полная стартовая конфигурация двухступенчатой космической транспортной системы Space Shuttle 
включает два боковых твердотопливных ракетных ускорителя, внешний топливный бак и орбитальный ракетоплан.

\section{1. Компьютерная поверхность твердотопливного ускорителя}

При запуске космической системы Space Shuttle пара боковых твердотопливных ускорителей (Solid Rocket Booster, SRB) создают более 80\% стартовой тяги (около $12.5 \mathrm{MH}$ каждый), которая плавно возрастает до максимума $(13.7 \mathrm{MH})$ в течение первых 20 с полета (табл.1). Остальную тягу производят собственные маршевые двигатели орбитальной ступени. Команда на зажигание ускорителей дается после подтверждения штатной работы основных двигателей орбитера, так как остановить процесс горения в твердотопливных двигателях невозможно. Ускорители продолжают работу в течение 122 с после старта, обеспечивая отрыв системы Space Shuttle от мобильной пусковой платформы и подъем на высоту около 45 км, затем отделяются. Ракеты-носители продолжают движение по инерции, через 75 с самостоятельного полета достигают максимальной высоты (порядка 65 км) и с помощью парашютной системы, срабатывающей по сигналу бародатчика, плавно приводняются в океане на расстоянии 230 км от места старта. Таким образом, конструкцией первой ступени системы Space Shuttle было предусмотрено ее повторное использование после ремонта и перезаправки $[2,3,31,32]$.

\section{Таблица 1}

Геометрические данные и энерго-массовые характеристики первой ступени космической системы Space Shuttle - боковых ускорителей $[2,3,31,32]$

\begin{tabular}{|l|c|}
\hline Характеристики & $\begin{array}{l}\text { Многоразовый твердотопливный } \\
\text { ракетный ускоритель (ТТРУ) }\end{array}$ \\
\hline Тип & 2 \\
\hline Количество & $\begin{array}{c}1400000 / 1275000 \\
(13734 / 12500)\end{array}$ \\
\hline $\begin{array}{l}\text { Максимальная / номинальная стартовая тяга } \\
\text { (одного ускорителя), кгс (кН) }\end{array}$ & 1300 \\
\hline Суммарный импульс, МН·с & 122 \\
\hline Продолжительность работы, с & 45 \\
\hline Высота отделения, км & \\
\hline Геометрические данные & 45.46 \\
\hline Длина, м & 3.71 \\
\hline Диаметр, м \\
\hline Массовые данные \\
\hline Стартовая масса (одного ускорителя), т & 589 \\
\hline Масса пустого ускорителя, т & 87 \\
\hline Масса топлива, т & 502 \\
\hline
\end{tabular}

Подъем кораблей Space Shuttle осуществлялся с помощью самых мощных твердотопливных ракетных ускорителей из когда-либо созданных.

Для конфигурации первой ступени космического транспортного корабля Space Shuttle с использованием современной системы автоматизированного проектирования SolidWorks [10] была построена сложная виртуальная поверхность (рис. 2). Созданная геометрическая модель многоразового твердотопливного ракетного ускорителя (ТТРУ) отражает все основные особенности конфигурации соответствующего прообраза. Габаритные размеры каждой ракеты-носителя, а также энерго-массовые характеристики представлены в табл.1.

Каждый ТТРУ имеет длину 45.46 м и диаметр 3.71 м, а основную часть его взлетной массы 589 т, составляет масса смесевого твердого топлива (502 т). Ракетный ускоритель представляет собой сборку из четырех однотипных сегментов (переднего, переднего центрального, хвостового центрального и хвостового). 


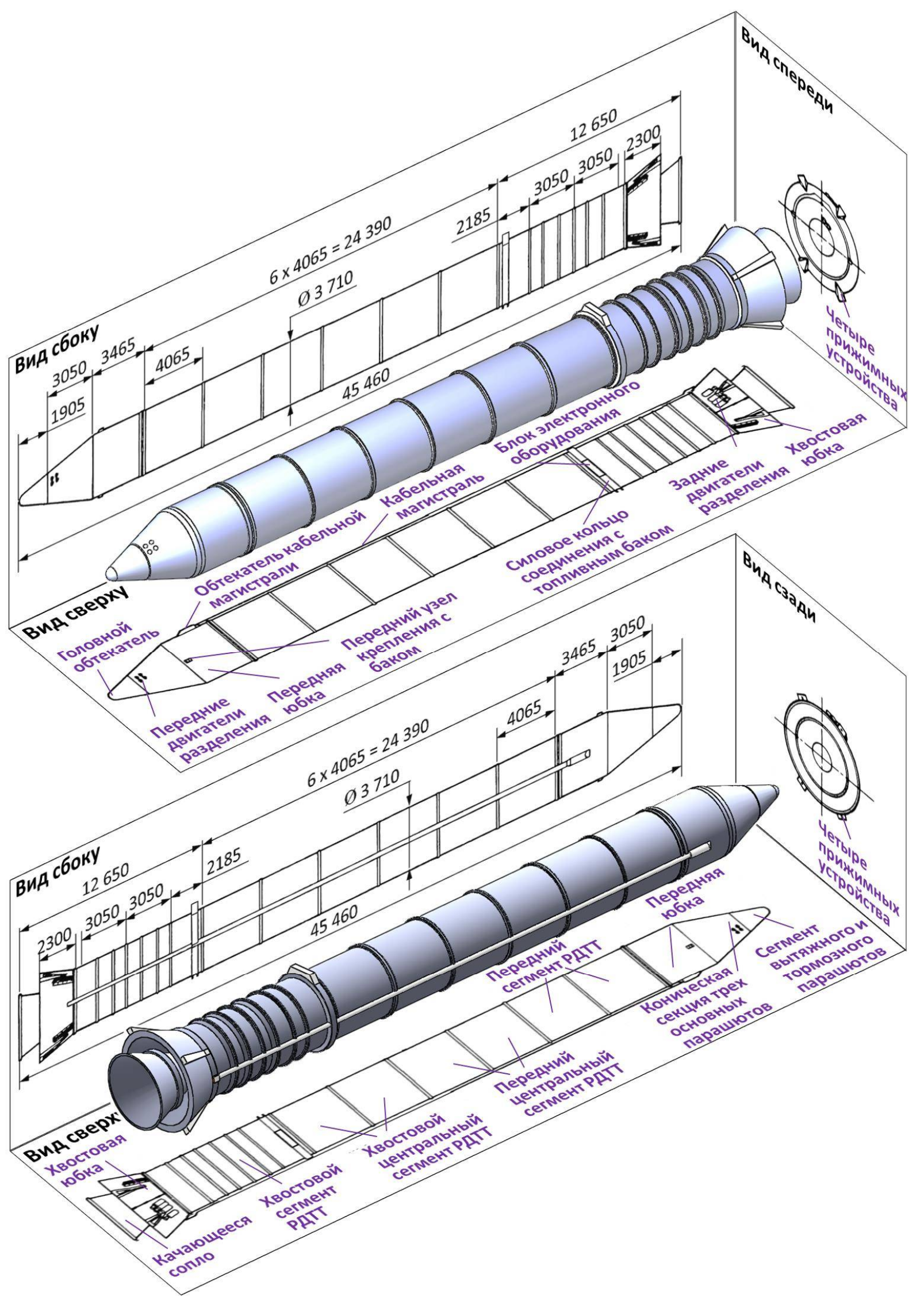

Рис. 2. Созданная виртуальная 3D поверхность боковых ускорителей многоразовой транспортной космической системы Space Shuttle 
Каждая секция представляет собой пару соединенных ракетных двигателей твердого топлива (РДТТ). Хвостовой сегмент оснащен качающимся соплом, которое отклоняется в диапазоне $\pm 8^{0}$, обеспечивая управление вектором тяги. Также к последней секции крепится нижняя (хвостовая) юбка на которой расположены задние двигатели отделения от внешнего топливного бака. На хвостовой юбке имеются четыре прижимных устройства (четыре пироболта), которые удерживают ускоритель на пусковой платформе и разрушаются при старте. К переднему сегменту РДТТ присоединяется верхняя (передняя) юбка. Последняя представляет собой проставку цилиндрической формы, которая придает плавучесть всей конструкции после приводнения. На переднюю юбку устанавливается головной обтекатель, разделенный на две секции - сегмент вытяжного и тормозного парашютов, а также конический сегмент трех основных парашютов. На носовом обтекателе располагаются четыре передних двигателя отделения от подвесного топливного отсека.

Для скрепления элементов конструкции многоразовой ракеты-носителя между собой используются хомуты, поэтому торцы каждого отсека снабжены кольцевыми выступами. Соединения герметизируется уплотнительными кольцами.

Снизу каждый ускоритель присоединяется к внешнему топливному баку с помощью силового кольца (основной узел крепления). Верхний узел крепления с топливным баком (вспомогательный) располагается на передней юбке.

\section{2. Геометрическая модель подвесного топливного отсека}

Внешний топливный бак $[2,3,33]$ содержит горючее (водород) и окислитель (кислород) для подпитки собственных разгонных двигателей орбитального ракетоплана. Топливо, размещенное в подвесном отсеке, обеспечивает бесперебойное функционирование маршевых силовых установок второй ступени на протяжении всего процесса вывода на орбиту. Работа двигателей орбитера начинается за 7 секунд до момента отрыва от стартового стола, и прекращается после 8 минут полета на высоте 105 км. После расходования запасов топлива внешний бак отбрасывается через 8.5 минут после старта на высоте 113 км и сгорает в атмосфере. В отдаленной перспективе планировалось обеспечить спасение дорогостоящей конструкции топливного бака, однако эта идея не была реализована.

Воссозданная в настоящей работе виртуальная модель внешнего топливного бака (рис. 3) детально отражает специфику геометрии и содержит все основные конструктивные элементы реального изделия.

Топливный бак монококовой конструкции, длина которого составляет 47 м, а диаметр - 8.38 м, состоит из трех сегментов. Верхняя секция (бак окислителя объемом 550 тыс. литров) предназначена для жидкого кислорода, поддерживаемого при температуре $-183{ }^{\circ} \mathrm{C}$. Бак окислителя имеет оживальную форму и эллипсоидальное нижнее днище. Нижний отсек (бак горючего) вмещает 1.45 млн. литров жидкого водорода температурой $-253{ }^{\circ} \mathrm{C}$ и занимает две трети всего полезного объема топливного бака. Бак горючего собран из цилиндрической обечайки, верхнего эллипсоидального днища и нижнего эллипсоидального днища. Топливные сегменты разделены цилиндрическим межбаковым отсеком длиной 7 м, на котором с двух диаметрально противоположных сторон располагаются передние узлы крепления боковых ускорителей. Конструкция промежуточной секции усилена большим количеством (более сотни) стрингеров, которые представляют собой продольные ребра на внешней стороне топливного бака. Силовой набор виртуальной поверхности топливного бака, представленной на рис. 3, включает 120 таких ребер.

Конструкция топливного бака изготавливалась из алюминиево-литиевого сплава. Термоизоляция, наносимая методом напыления на внешнюю поверхность бака, предотвращала его обледенение, а также защищала кислород и водород от перегрева. Для поддержания оптимальной температуры сжиженных компонентов топлива использовалась сложная система кондиционирования. 


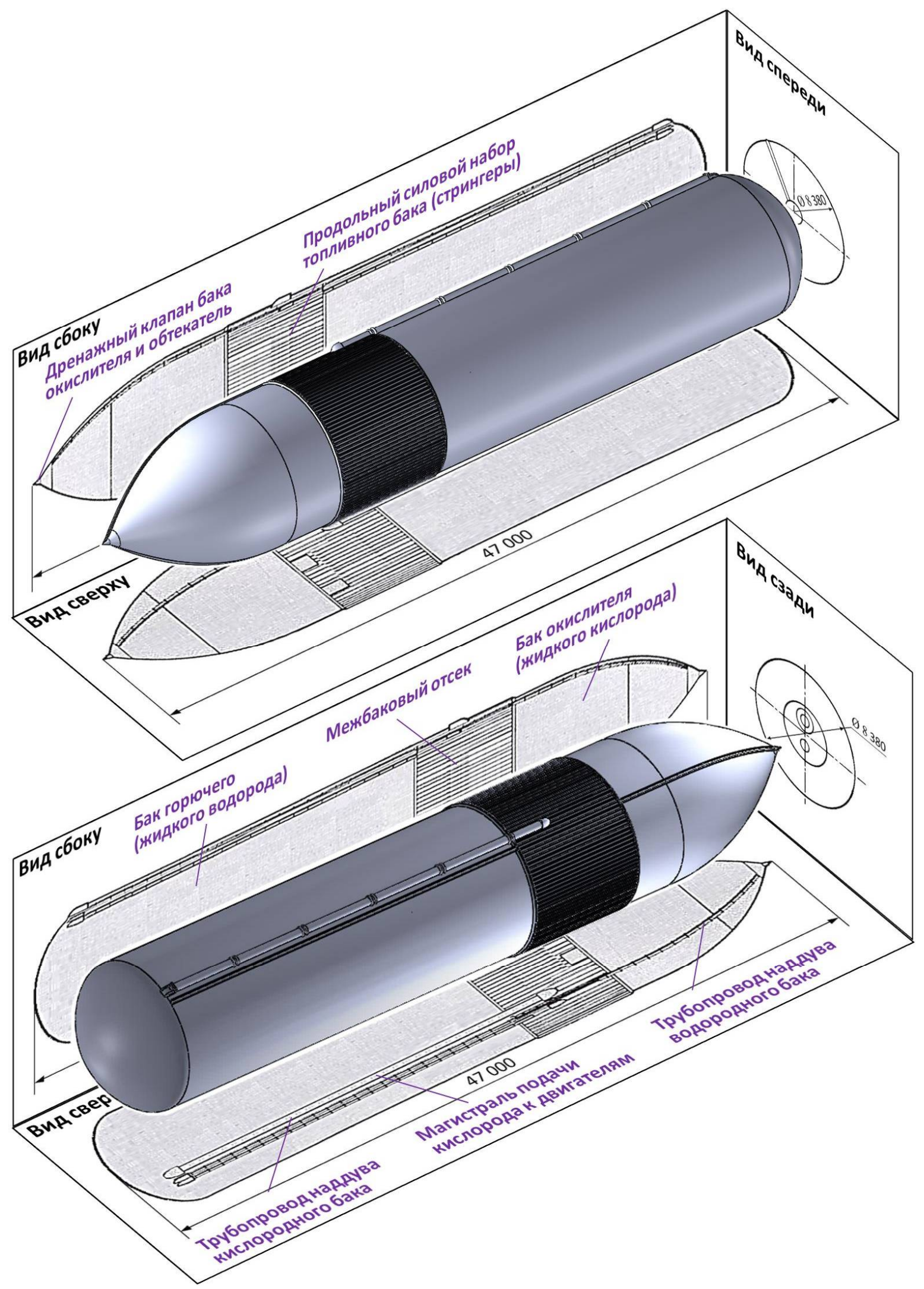

Рис. 3. Реалистичная компьютерная поверхность внешнего топливного бака второй ступени космической системы Space Shuttle

Подвесной топливный отсек оснащался системами дренажа и наддува, с помощью которых осуществлялась регулировка давления в топливных резервуарах, а также системой защиты от атмосферного электричества (молний). Состояние работы систем контролировалось 
с помощью множества датчиков. Горючее и окислитель из бака подводились к трем маршевым двигателям орбитальной ступени по топливным магистралям.

Основные элементы перечисленных систем, определяющие внешнюю конфигурацию топливного бака, были учтены при создании виртуальной поверхности последнего (рис. 3). В частности, компьютерная модель рассматриваемого объекта оснащена обтекателем со встроенным дренажным клапаном кислородного бака, трубопроводами наддува и магистралью подачи окислителя. Основные геометрические и массовые характеристики внешнего топливного бака помещены в табл. 2.

\section{Таблица 2}

Геометрические и массовые характеристики подвесного топливного отсека второй ступени космической системы Space Shuttle $[2,3,33]$

\begin{tabular}{|l|c|}
\hline Характеристики & $\begin{array}{c}\text { Одноразовый внешний } \\
\text { топливный бак }\end{array}$ \\
\hline Тип & 1 \\
\hline Количество & 8.5 \\
\hline Время отделения после старта, мин & 113 \\
\hline Высота отделения, км & 47 \\
\hline Геометрические данные & 8.38 \\
\hline Длина, м & 550 \\
\hline Диаметр, м & 1450 \\
\hline Внутренний полезный объем бака окислителя, ${ }^{3}$ & \\
\hline Внутренний полезный объем бака горючего, ${ }^{3}$ & 740 \\
\hline Массовые данные & 33.5 \\
\hline Стартовая масса, т & 102 \\
\hline Сухая масса, т & 0.23 \\
\hline Масса горючего (жидкий водород), т & 604 \\
\hline Давление наддува бака горючего, МПа & 0.15 \\
\hline Масса окислителя (жидкий кислород), т & \\
\hline Давление наддува бака окислителя, МПа & \\
\hline
\end{tabular}

\section{3. Виртуальная модель поверхности орбитальной ступени}

Пилотируемый орбитальный корабль системы Space Shuttle представляет собой воздушно-космический самолет с низкорасположенным дельтавидным крылом [2,3,24-30]. Его длина составляет 37.24 м (табл. 3, рис. 4), максимальная высота по килю - 17.25 м, а размах крыла с двойной стреловидностью - 23.84 м (угол стреловидности корневой части консоли равен $81^{\circ}$, концевой части $-45^{\circ}$ ).

Ракетоплан оснащен тремя собственными маршевыми жидкостными ракетными двигателями (ЖРД) Rocketdyne (RS-25), которые создают дополнительный разгонный импульс при выведении на орбиту. Главные силовые установки орбитера, которые подпитываются жидким водородом и кислородом из большого подвесного топливного бака, прекращают работу на высоте 105 км. Через 90 секунд после отделения топливного отсека, разгонный импульс, необходимый для выхода на устойчивую низкую круговую орбиту, выдается двигателями системы орбитального маневрирования (Orbital Maneuvering System, OMS). Также два двигателя OMS используются для совершения маневров на орбите и схода с нее. Для точного задания пространственного положения орбитальной ступени применяется система двигателей ориентации (реактивной системы управления, PCУ). Система PCУ (Reaction Control System, RCS) состоит из 44-х жидкостных ракетных двигателей малой тяги. Передний ряд из 16-ти силовых установок размещается в носовой части корпуса. Задний ряд включает 28 ЖРД и располагается в блоках двигателей орбитального маневрирования (по 14 в каждом блоке). 


\section{Таблища 3}

Геометрические данные и энерго-массовые характеристики орбитального ракетоплана космической системы Space Shuttle [2, 3, 24-30, 35-37]

\begin{tabular}{|c|c|}
\hline \multicolumn{2}{|l|}{ Характеристики } \\
\hline Тип главных маршевых двигателей & $\begin{array}{l}\text { Жидкостные ракетные дви- } \\
\text { гатели Rocketdyne (RS-25) }\end{array}$ \\
\hline Количество главных двигателей & 3 \\
\hline $\begin{array}{l}\text { Номинальная стартовая тяга (одной силовой } \\
\text { установки), кгс (кН) }\end{array}$ & $190000(1860)$ \\
\hline $\begin{array}{l}\text { Продолжительность работы основных двигате- } \\
\text { лей после старта, мин }\end{array}$ & 8 \\
\hline Высота отключения, км & 105 \\
\hline $\begin{array}{l}\text { Тип двигателей системы орбитального маневри- } \\
\text { рования (OMS) }\end{array}$ & $\begin{array}{l}\text { Жидкостные ракетные дви- } \\
\text { гатели AJ10-190 }\end{array}$ \\
\hline Количество двигателей OMS & 2 \\
\hline $\begin{array}{l}\text { Тяга двигателей орбитального маневрирования } \\
\text { (одной силовой установки), кН }\end{array}$ & 26.7 \\
\hline $\begin{array}{l}\text { Тип двигателей ориентации (реактивного управ- } \\
\text { ления) }\end{array}$ & $\begin{array}{l}\text { Жидкостные ракетные дви- } \\
\text { гатели малой тяги }\end{array}$ \\
\hline $\begin{array}{l}\text { Количество двигателей реактивной системы } \\
\text { управления (RCS) }\end{array}$ & 44 \\
\hline $\begin{array}{l}\text { Тяга двигателей PCУ/RCS (одной силовой уста- } \\
\text { новки), кН }\end{array}$ & 0.387 \\
\hline \multicolumn{2}{|l|}{ Геометрические и массовые данные } \\
\hline Масса (без полезного груза), т & 80.0 \\
\hline Максимальная стартовая масса, т & 104.0 \\
\hline Максимальный линейный размер $L_{\max }$, м & 37.238 \\
\hline Характерная длина $L_{\mathrm{ref}}, \mathrm{м}$ & 32.770 \\
\hline Размах крыла $b$, м & 23.842 \\
\hline Высота, м & 14.120 \\
\hline Характерная площадь $S_{\text {ref, }}{ }^{2}$ & 249.909 \\
\hline Средняя аэродинамическая хорда $c$, м & 12.060 \\
\hline \multicolumn{2}{|l|}{ Расположение центра тяжести } \\
\hline - расстояние от носа вдоль оси $x$, м & $21.303\left(0.65 L_{\mathrm{ref}}\right)$ \\
\hline - расстояние от верхушки киля вдоль оси $y$, м & 11.270 \\
\hline
\end{tabular}

Аэродинамическая конфигурация крылатой орбитальной ступени обеспечивает оптимальные несущие свойства и приемлемые тепловые нагрузки на любом участке планирующей траектории спуска в атмосфере $[26,27,30,34]$ в широком диапазоне скоростей и углов атаки. Орбитальный самолет начинает вход в атмосферу с углом атаки $40^{\circ}$ и скоростью 7.8 км/с $(\mathrm{M}=25)$. Пока основная часть орбитальной скорости не будет погашена, ракетоплан сохраняет большие углы атаки $\alpha$, затем продолжает снижаться как тяжелый планер с невысоким аэродинамическим качеством, постепенно уменьшая угол атаки. На траекторном участке, соответствующем значительным сверхзвуковым скоростям от $\mathrm{M}=20\left(\alpha=40^{0}\right)$ до $\mathrm{M}=10\left(\alpha=35^{0}\right)$, аэродинамическое качество орбитального самолета возрастает от $k=1.1$ до $k=1.25$. В диапазоне умеренных скоростей $(\mathrm{M}=4 \div 8)$ при уменьшении числа Маха и угла атаки с $\alpha=30^{0}$ до $\alpha=15^{0}$ качество увеличивается до $k=1.9 \div 2.0$. Для трансзвукового участка характерны значения $k=1.8 \div 1.9$. На заключительном этапе спуска при переходе на дозвуковой режим (для $\alpha=5^{0} \div 10^{0}$ ) отношение подъемной силы к лобовому сопротивлению поддерживается около значения $k \approx 3.5$. 


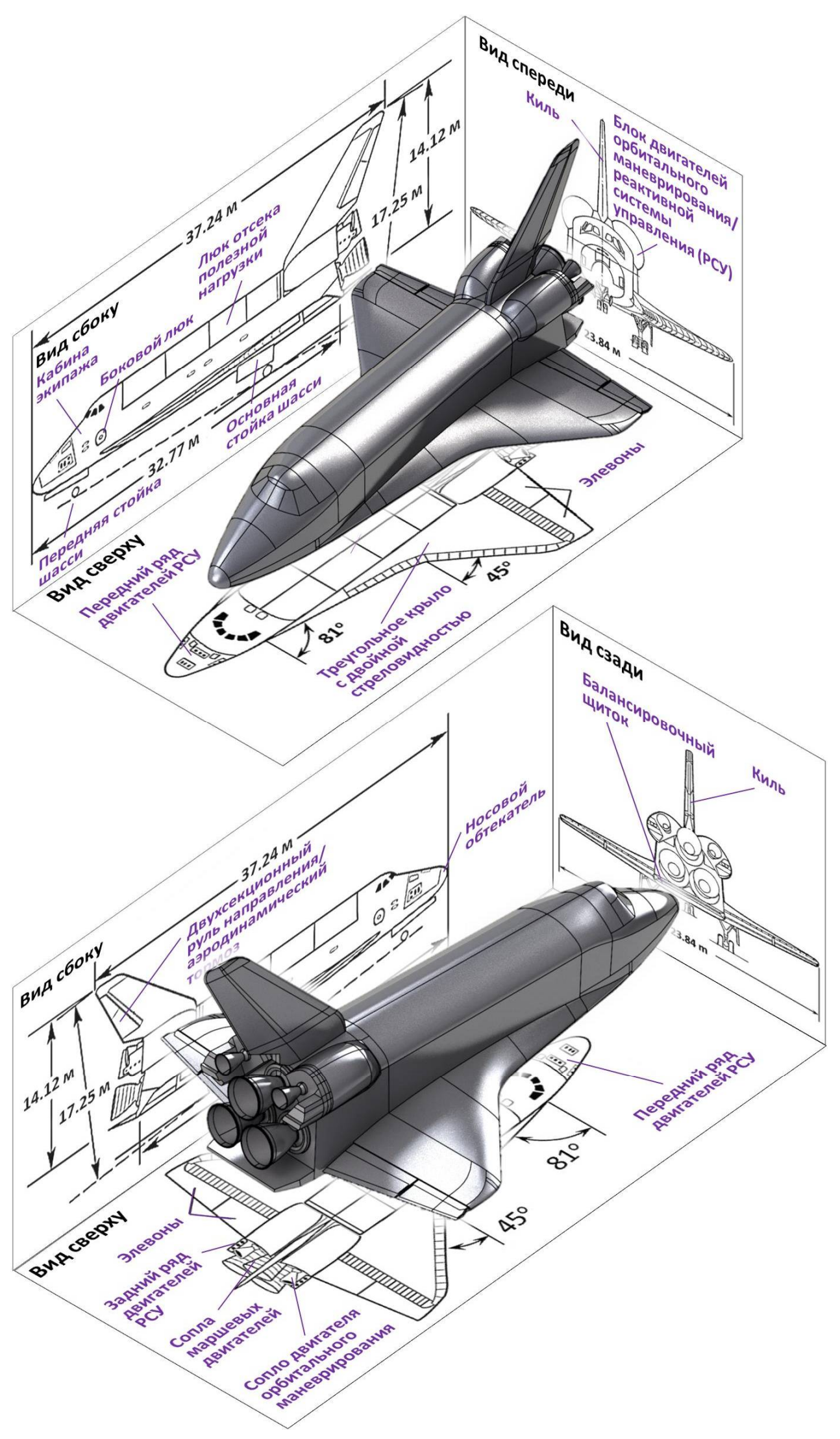

Рис. 4. Созданная виртуальная 3D поверхность орбитальной ступени многоразовой космической транспортной системы Space Shuttle Atlantis

Стабилизация летательного аппарата и маневрирование при движении в атмосфере осуществляются с помощью отклоняемых аэродинамических поверхностей и реактивной системы управления. 
Уже через 140 с после входа в атмосферу (на высоте 100 км) становится возможной балансировка орбитера с использованием управляющих поверхностей. На высоте 95 км (через 180 с после входа) космоплан переходит к совместному использованию РСУ и средств механизации крыла. На 280-й секунде (высота 84 км) ракетные двигатели системы управления отключаются.

Механизация треугольного крыла с двойной стреловидностью включает элевоны, расположенные на задних кромках. Элевоны обеспечивают крен (при несимметричном отклонении), а также могут работать в режиме руля высоты. Подфюзеляжный (балансировочный) щиток, расположенный в задней части орбитального самолета под хвостовой частью фюзеляжа, обеспечивает балансировку, а также используется для помощи в совершении маневров по тангажу. Кроме этого, для эффективного пилотирования в плотных слоях атмосферы используется двухсекционный руль направления на киле. Последний служит также воздушным тормозом, предназначенным для увеличения сопротивления движению на заключительных стадиях - при заходе на посадку и приземлении.

Сложная виртуальная поверхность, созданная для исследуемой конфигурации орбитальной ступени космической транспортной системы Space Shuttle, представлена на рис.4. Геометрическая модель учитывает специфические особенности орбитального самолета, и содержит все основные конструктивные элементы соответствующего прообраза. Габаритные размеры второй ступени многоразового космического корабля Space Shuttle, а также некоторые массовые характеристики орбитера представлены в табл.3.

Виртуальная модель включает двигатели системы орбитального маневрирования, внутренние и внешние поверхности сопел трех маршевых двигателей, киль, двухсекционный руль направления, треугольное крыло с двойной стреловидностью, отклоняемые управляющие поверхности крыла (элевоны), подфюзеляжный (балансировочный) щиток, расположенный в задней части орбитального самолета под хвостовой частью фюзеляжа. Модель предельно точно отражает конфигурации днища, носовой и хвостовой частей корпуса, форму крыла.

\section{4. Копмьютерная модель поверхности полной компоновки многоразового транспортного космического корабля Space Shuttle во взлетной конфигурации}

Space Shuttle - самый большой транспортный космический корабль из когда-либо построенных. Высота его полной компоновки в стартовой конфигурация составляет 56.14 м (табл. 4 , рис. 5,6 ).

\section{Таблийа 4}

Геометрические данные и энерго-массовые характеристики космической системы Space Shuttle

\begin{tabular}{|c|c|}
\hline \multicolumn{2}{|c|}{ Геометрические данные всей системы } \\
\hline Высота на стартовой позиции, м & 56.14 \\
\hline \multicolumn{2}{|c|}{ Энерго-массовые характеристики всей системы } \\
\hline Общая стартовая масса, т & 2020 \\
\hline $\begin{array}{l}\text { Масса полезной нагрузки, т } \\
\text { - вывод на низкую опорную орбиту, т } \\
\text { - вывод на геостационарную орбиту, т }\end{array}$ & $\begin{array}{l}24.4 \\
3.8\end{array}$ \\
\hline $\begin{array}{l}\text { Максимальная масса возвращаемого с орбиты } \\
\text { полезного груза, т }\end{array}$ & 14.5 \\
\hline Полная стартовая тяга системы, кН & 30580 \\
\hline
\end{tabular}

Концептуально космический транспортный корабль Space Shuttle представляет собой выводимый на орбиту летательный аппарат с полезной нагрузкой (экипажем, оборудованием и т.п.) и двигательную систему, обеспечивающую его ускорение. Энергетическая система Space Shuttle включает два боковых твердотопливных ускорителя, подвесной топливный бак, 
а также собственные кислородно-водородные силовые установки орбитального ракетоплана и обеспечивает стартовую тягу 30580 кН. Запуск космической системы выполняется на полной тяге собственных маршевых двигателей шаттла и двух боковых твердотопливных ускорителей. При этом ускорители отделяются после двух минут полета, топливный бак - через 8.5 минут. Главные силовые установки и твердотопливные ускорители после ремонта используются повторно, а внешний топливный отсек сгорает в атмосфере.

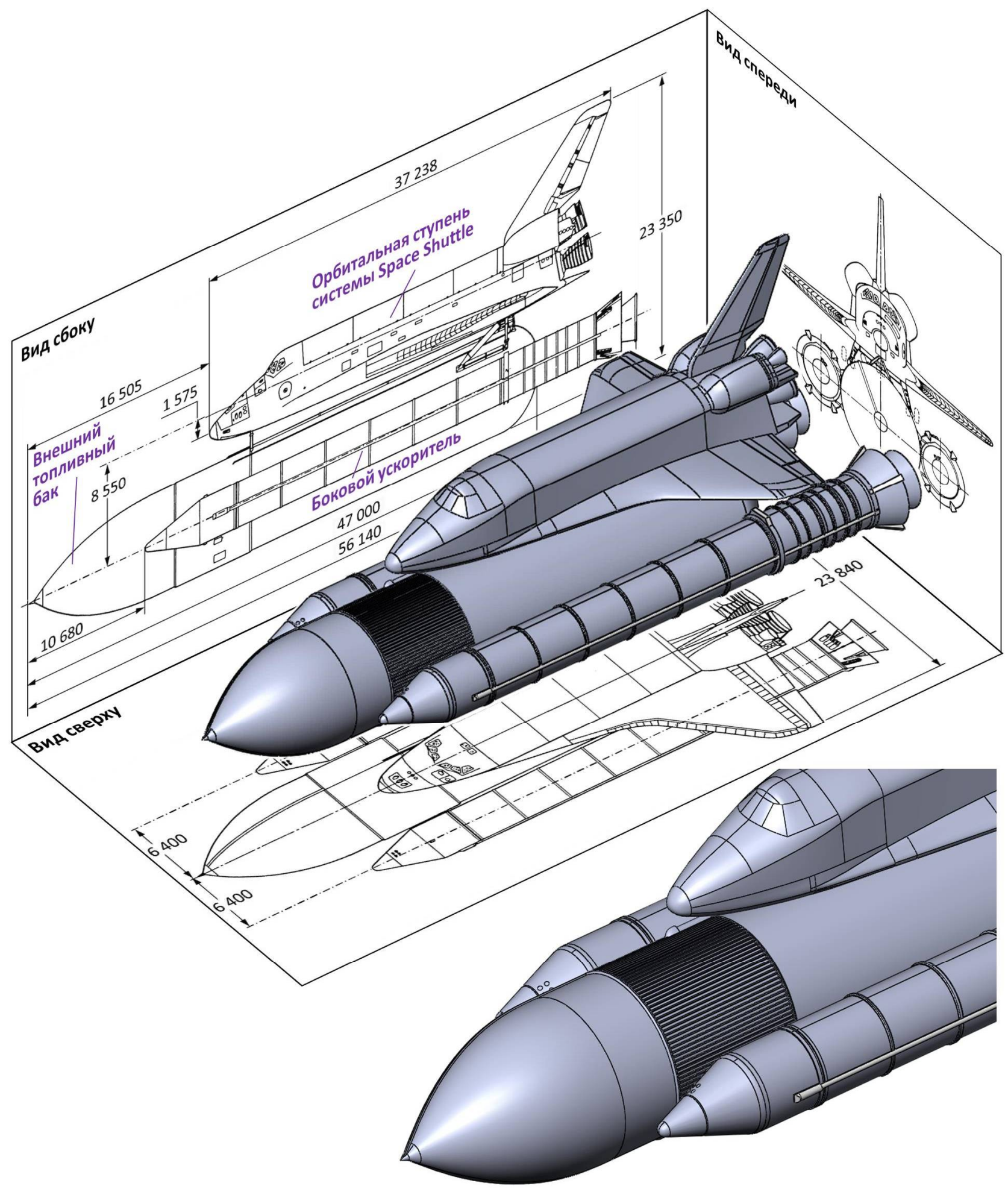

Рис. 5. Компьютерная поверхность многоразовой транспортной космической системы Space Shuttle во взлетной конфигурации. Вид спереди 


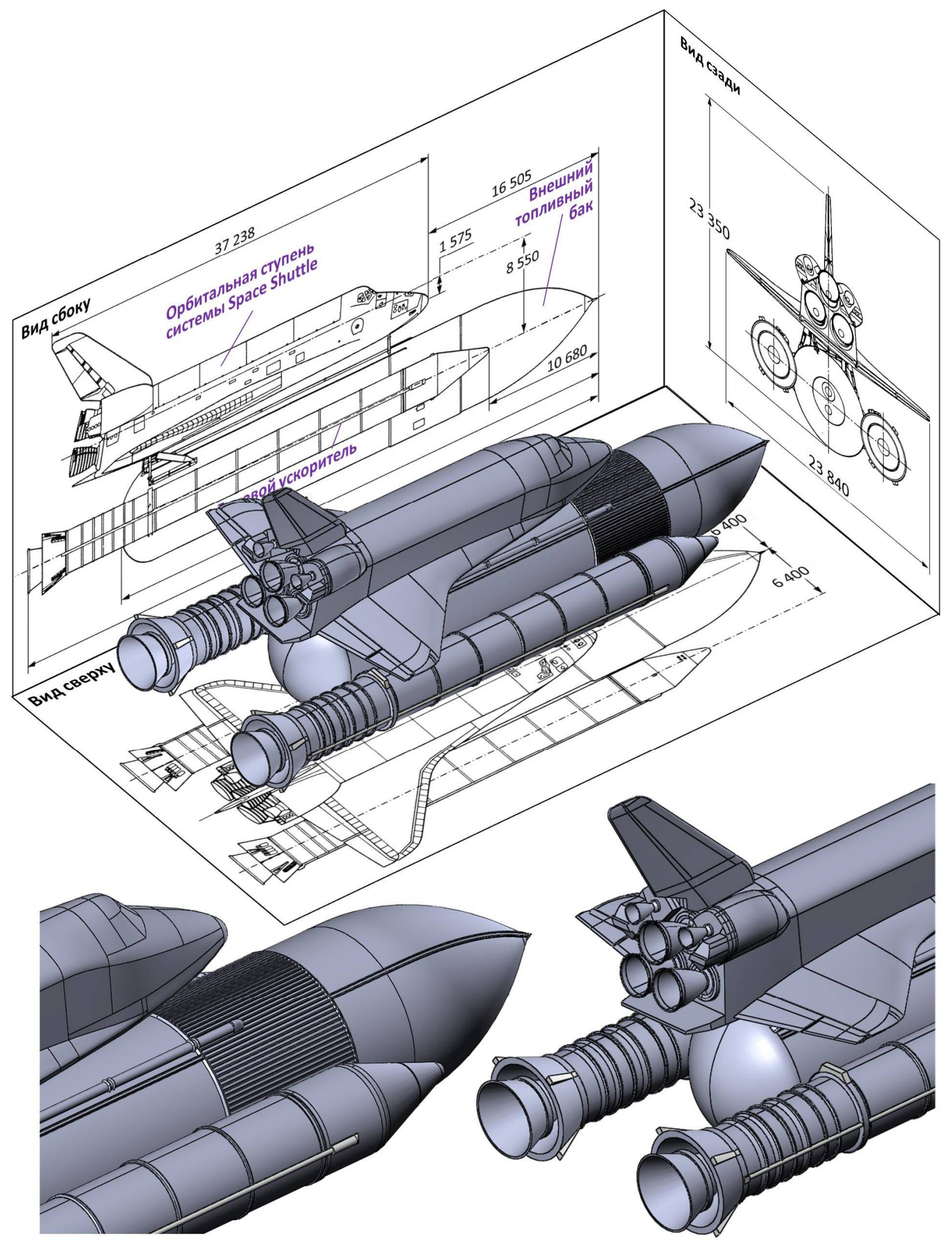

Рис. 6. Компьютерная поверхность многоразовой транспортной космической системы Space Shuttle во взлетной конфигурации. Вид сзади

Фактически корабль Space Shuttle можно считать, следуя терминологии [38], “полутораступенчатым", так как в процессе ускорения одновременно функционируют все элементы 
энергетической системы, а орбитальная скорость достигалась при непрерывной работе главных двигателей космолета. В конструкции Space Shuttle была впервые реализована перспективная схема с боковым креплением ускорителей, что потребовало значительных усилий по интеграции многомодульной аэродинамической компоновки. На начальных этапах проектирования и создания концепции космической системы ее технический облик претерпевал значительные изменения [38]. Решение использовать сбрасываемый внешний топливный бак ощутимо снизило стоимость разработки изделия. Первоначальный проект, предполагавший применение многоразовых жидкостных ракетных систем в качестве первой ступени, был пересмотрен в пользу восстанавливаемых двигателей твердого топлива (РДТТ). До этого времени инженеры NASA не имели опыта эксплуатации РДТТ в ходе выполнения своих космических программ (твердотопливные установки создавались исключительно для нужд ВВС США).

Уникальные размеры ускорителей, жесткое ограничение по перегрузкам для пилотируемых миссий и обеспечение возможности повторного использования усложняли поставленную задачу. Конфигурация космической транспортной системы также формировалась с учетом интересов оборонной отрасли. В частности, от космического транспортного средства для доставки полезных грузов на орбиту требовалось выполнение полярных орбитальных миссий (с углом наклонения орбиты $90^{\circ}$ ) и быстрое возвращение на Землю с высокой точностью приземления на заданной посадочной площадке. Последнее привело к утверждению концепции дельтовидного крыла для конструкции орбитального самолета. Размер системы и производительность силовых установок определялись исходя из задач вывода значительной полезной нагрузки. Таким образом, окончательные схемно-конструктивные решения принимались под влиянием указанных факторов, в рамках установленных предельно-допустимых затрат.

Несмотря на тщательную проектную проработку каждого элемента системы, существенные изменения в конструкцию приходилось вносить на последнем этапе комплексных испытаний и в процессе эксплуатации. В частности, выяснилось, что отклоняемые сопла главных маршевых двигателей не могли обеспечить достаточную управляемость компоновки и изначально неподвижные сопла твердотопливных ракетных ускорителей были заменены на поворотные. Последнее обстоятельство подчеркивает важность компьютерной поддержки в проведении работ по интеграции многокомпонентных аэрокосмических систем.

Рисунки 5, 6 дают представление об особенностях конструктивного исполнения и взаимного расположения ступеней космической транспортной системы Space Shuttle, самым большим элементом которой является внешний топливный бак. Подвесной топливный отсек - основной связующий и силовой элемент космического корабля. Он служит каркасом конструкции и воспринимает все нагрузки, передающиеся от боковых ускорителей и собственных разгонных двигателей орбитального ракетоплана.

\section{3. Сеточные модели}

На основе разработанных максимально реалистичных виртуальных поверхностей отдельных элементов космической транспортной системы Space Shuttle: твердотопливных ускорителей, топливного бака и орбитального ракетоплана (см. рис. 2-4) была сгенерирована серия качественных тетраэдральных расчетных сеток (независимо для каждого составляющего элемента космической системы). Качество сеточных моделей определялось по соответствию ряду общепринятых критериев $[39,40]$. Выбор неструктурированного подхода, был обусловлен высокой трудоемкостью построения блочно-структурированных сеточных моделей около рассматриваемой конфигурации, а также сложностью автоматизации процесса разбиения на подобласти и последующего сопряжения блоков. Используемые алгоритмы построения нерегулярных тетраэдральных сеток позволили обеспечить быстроту выполнения, надежность результатов при умеренных затратах вычислительных ресурсов и расчетного времени, максимальную гибкость, универсальность и адаптивность. Последнее является кри- 
тичным фактором при решении задач моделирования обтекания многоступенчатых космических систем в полной компоновке с изменяемым взаимным положением ступеней (например, в процессе их разделения). Использование набора перекрывающихся нерегулярных сеток для построения композитной сеточной модели, дают возможность дискретизировать расчетную область произвольной формы и относительно легко получать локальные сгущения узлов в зонах больших градиентов. Кроме этого, нет необходимости увеличивать число сеток с увеличением сложности геометрии.

Надежность полученных результатов моделирования достигается, в первую очередь, благодаря хорошему качеству дискретизации сложных поверхностей, которая сохраняет (насколько это возможно в рамках неструктурированного подхода) высокую точность воспроизведения исследуемых конфигураций, заложенную в созданных геометрических моделях.

Сеточные пространства создаются независимо около каждой ступени космического корабля, затем объединяются в рамках единой композитной сеточной модели. Предлагаемый подход не требует многократного перестроения расчетных сеток при изменении количества или взаимного положения компонентов системы совместно обтекаемых тел произвольной конфигурации. Последнее имеет исключительное значение, поскольку создание расчетной сетки в процессе моделирования систем такой сложности (даже при использовании неструктурированно подхода) может потребовать больше временных ресурсов, чем сам расчет.

Для разрешения разномасштабных элементов конструкции каждого модуля космической системы Space Shuttle поверхностные расчетные сетки значительно сгущаются около мелких конструктивных элементов и в окрестности острых и скругленных кромок. При этом плотность узлов объемных сеток существенно возрастает вблизи обтекаемых тел в пространстве расчетной области.

Несмотря на использование адаптивных сеток, учет большого числа деталей рассматриваемых конфигураций и всех основных особенностей соответствующих прообразов потребовал построения сеточных моделей размерностью $9 \div 12 \times 10^{6}$ тетраэдральных элементов.

При создании адаптивных неструктурированных сеток были соблюдены все общепринятые требования к их качеству по изотропности, значениям коэффициентов формы (аспектному соотношению) и асимметрии $[39,40]$ :

1) Соседние треугольные или тетраэдральные элементы отличаются по размерам не более чем на $20 \%$.

2) Аспектное соотношение (aspect ratio) для любой треугольной ячейки поверхностной сетки и тетраэдрального элемента объемной сетки не превышает 4 и 6 соответственно.

3) Коэффициент асимметрии (equiangle skew), характеризующий угловую скошенность, для треугольных элементов меньше 0.65, для тетраэдральных - меньше 0.7.

Вблизи носовых обтекателей, двигательных установок, мелких конструктивных элементов, кромок крыла и управляющих поверхностей (для модели орбитальной ступени) размер тетраэдров не превышает 0.003 м. Разрешающая способность построенных сеток обеспечивает возможность определения динамических характеристик обтекаемых поверхностей с учетом сжимаемости и аэродинамического нагрева с инженерной точностью. Построенные сеточные модели полностью удовлетворяют требованиям, выполнение которых необходимо для эффективной работы модифицированных в рамках предложенного подхода вычислительных кодов, которые реализуют интегрирование полной системы уравнений сплошной среды (уравнений Навье-Стокса).

В соответствии с излагаемым в настоящей статье подходом, для конфигурации внешнего топливного бака (см. рис. 3) - связующего элемента всей конструкции, была независимо сгенерирована адаптивная тетраэдральная расчетная сетка (рис. 7) размерностью порядка 9 млн элементов. Границы расчетной области определялись таким образом, чтобы охватить все возмущенное пространство в окрестности космической транспортной системы в полной компоновке. 


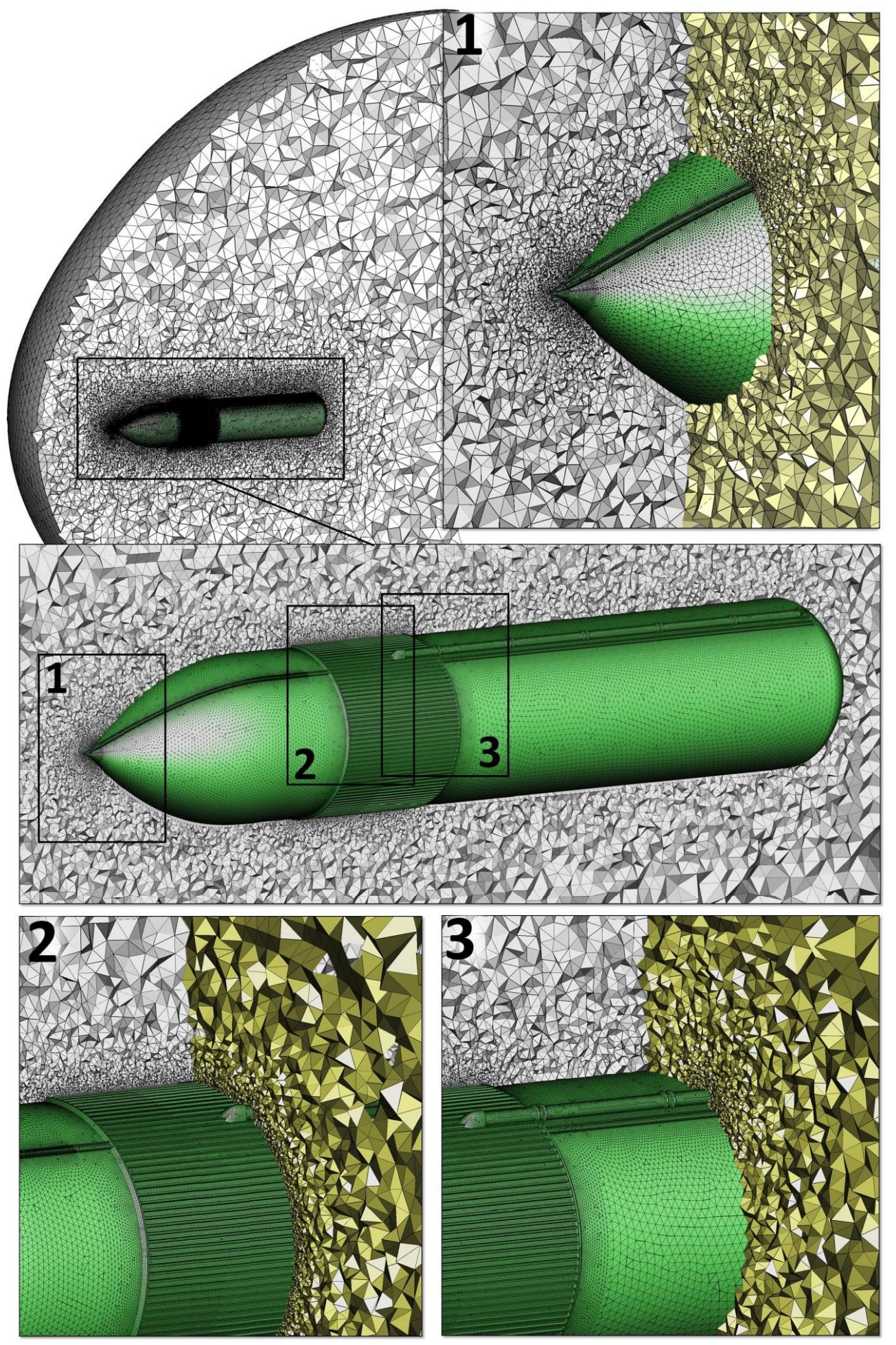

Рис. 7. Фрагменты объемной неструктурированной расчетной сетки размерностью 9035189 тетраэдров, сгенерированной около виртуальной модели топливного отсека и охватывающей всю возмущенную область течения

Для остальных компонентов космического корабля Space Shuttle: твердотопливного ускорителя (см. рис. 2) и орбитального ракетоплана $[6,34]$ (см. рис. 4) также генерировались отдельные сеточные модели с числом расчетных элементов около 10 и 12 млн соответственно. В отличие от сеточного пространства, построенного для топливного отсека, сетки орбитера и ускорителя покрывают только небольшую область в непосредственной близости от соответствующих конфигураций (рис. 8,9$)$. 


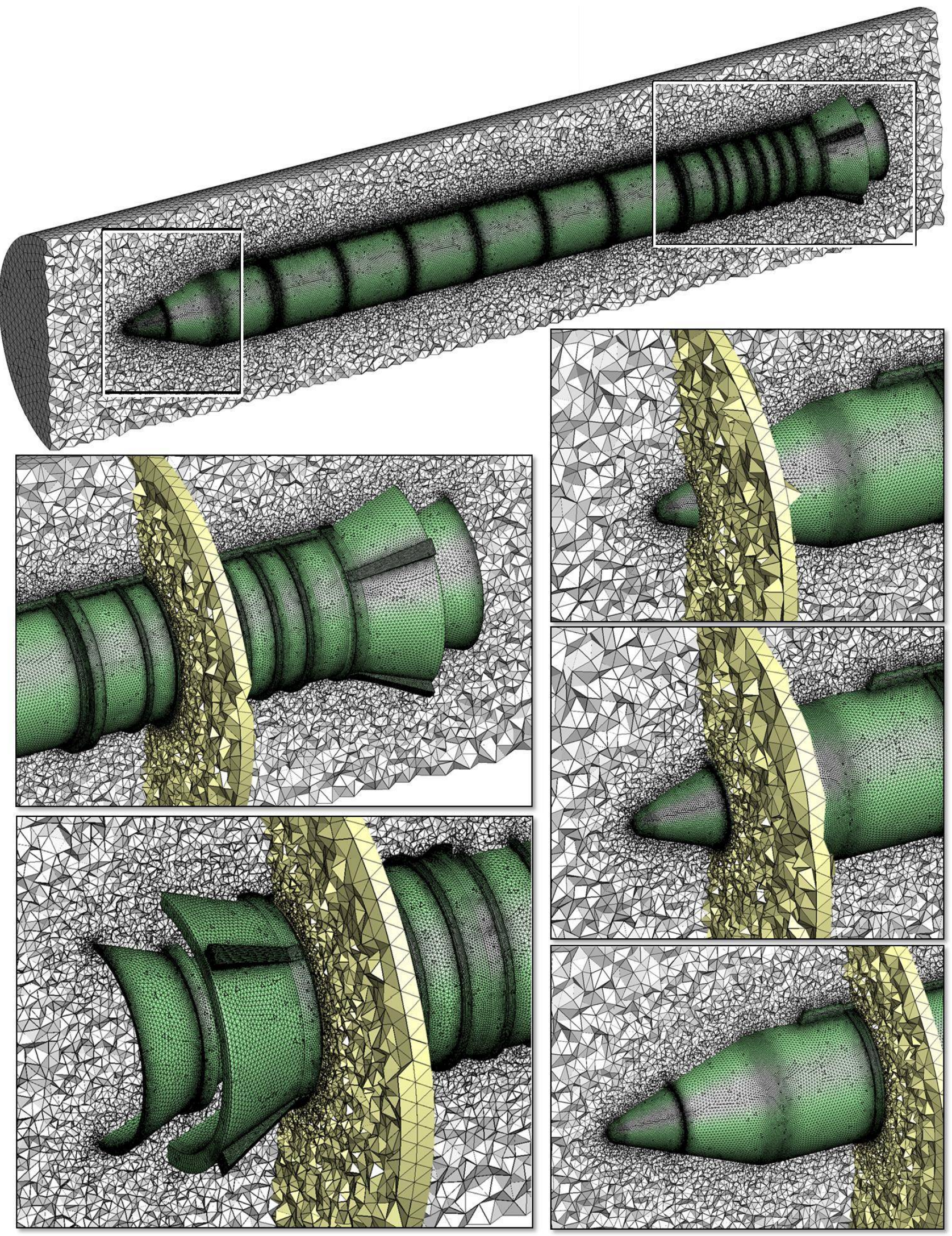

Рис. 8. Объемная неструктурированная сетка, независимо построенная около виртуального прототипа твердотопливного ускорителя системы Space Shuttle. Размерность сеточной модели 10280406 тетраэдральных ячеек 


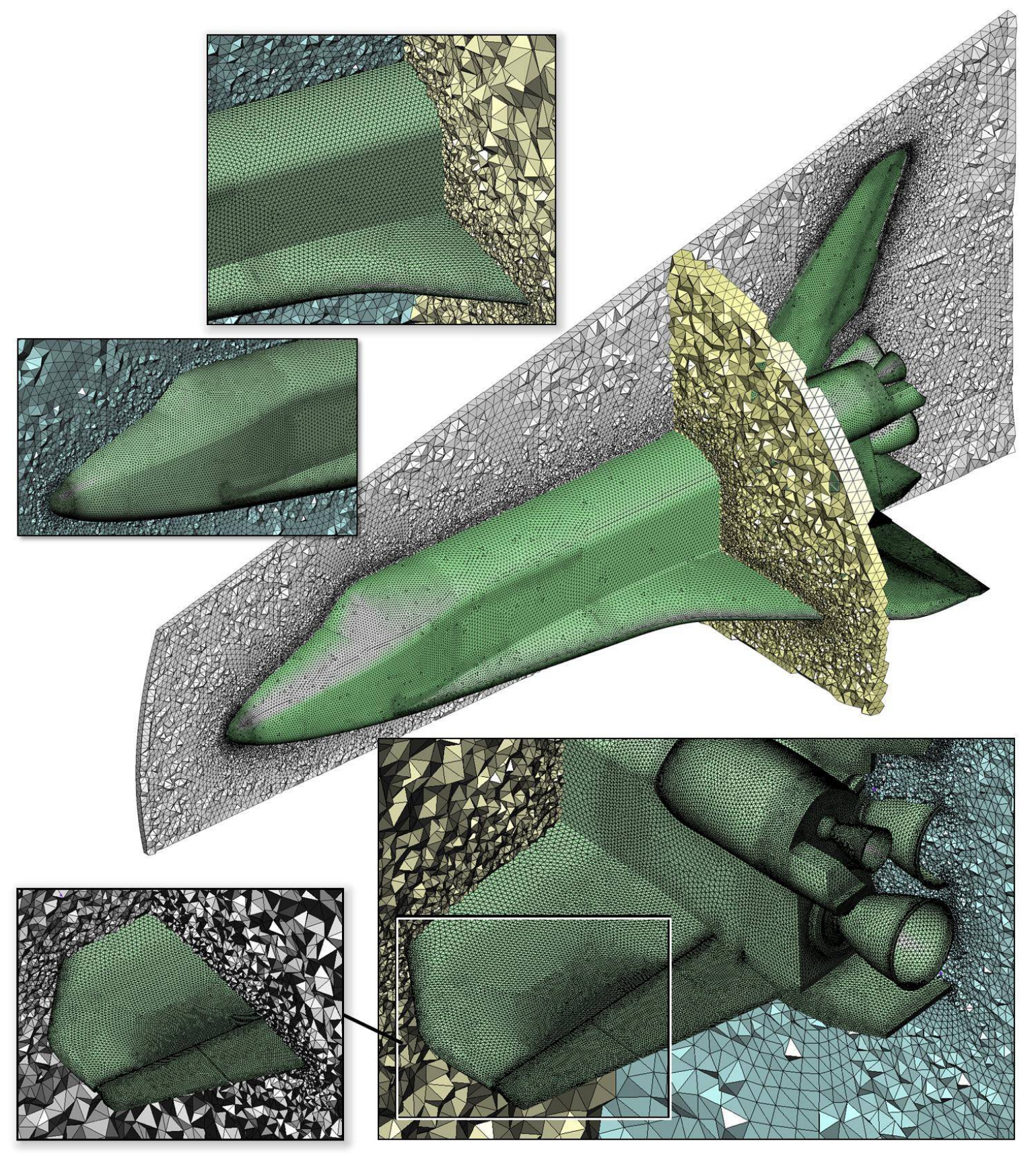

Рис.9. Треугольная сетка на поверхности орбитальной ступени системы Space Shuttle и несколько слоев тетраэдральных элементов в различных сечениях. Структура подробной тетраэдральной сетки размерностью 12665382 ячеек вблизи реалистичной конфигурации $[6,34]$

Некоторые характеристики разработанных сеточных моделей отдельных структурных элементов космического корабля (рис. 7-9) представлены в табл. 5.

Таблий 5

\section{Параметры созданных неструктурированных тетраэдральных расчетных сеток}

\begin{tabular}{|c|c|c|c|c|}
\hline \multirow{2}{*}{$\begin{array}{l}\text { Элемент } \\
\text { системы }\end{array}$} & \multicolumn{2}{|c|}{ Поверхностная сетка } & \multicolumn{2}{|c|}{ Объемная сетка } \\
\hline & Число узлов & Число треугольников & Число узлов & Число тетраэдров \\
\hline Топливный бак & 205693 & 411382 & 1613142 & 9035189 \\
\hline Ускоритель & 289118 & 577828 & 1865700 & 10280406 \\
\hline Орбитер & 155313 & 310368 & 2183325 & 12665382 \\
\hline
\end{tabular}




\section{4. Концепция создания композитной сеточной модели для многоступенчатой аэрокосмической системы}

Для объединения отдельных сеток, независимо сгенерированных для каждого модуля космической системы, в рамках единой композитной сеточной модели, используется метод перекрывающихся подобластей с неструктурированным сеточным разбиением (рис. 10). Представленная на рисунке составная модель соответствует взлетной конфигурации транспортного корабля Space Shuttle (см. рис. 6).
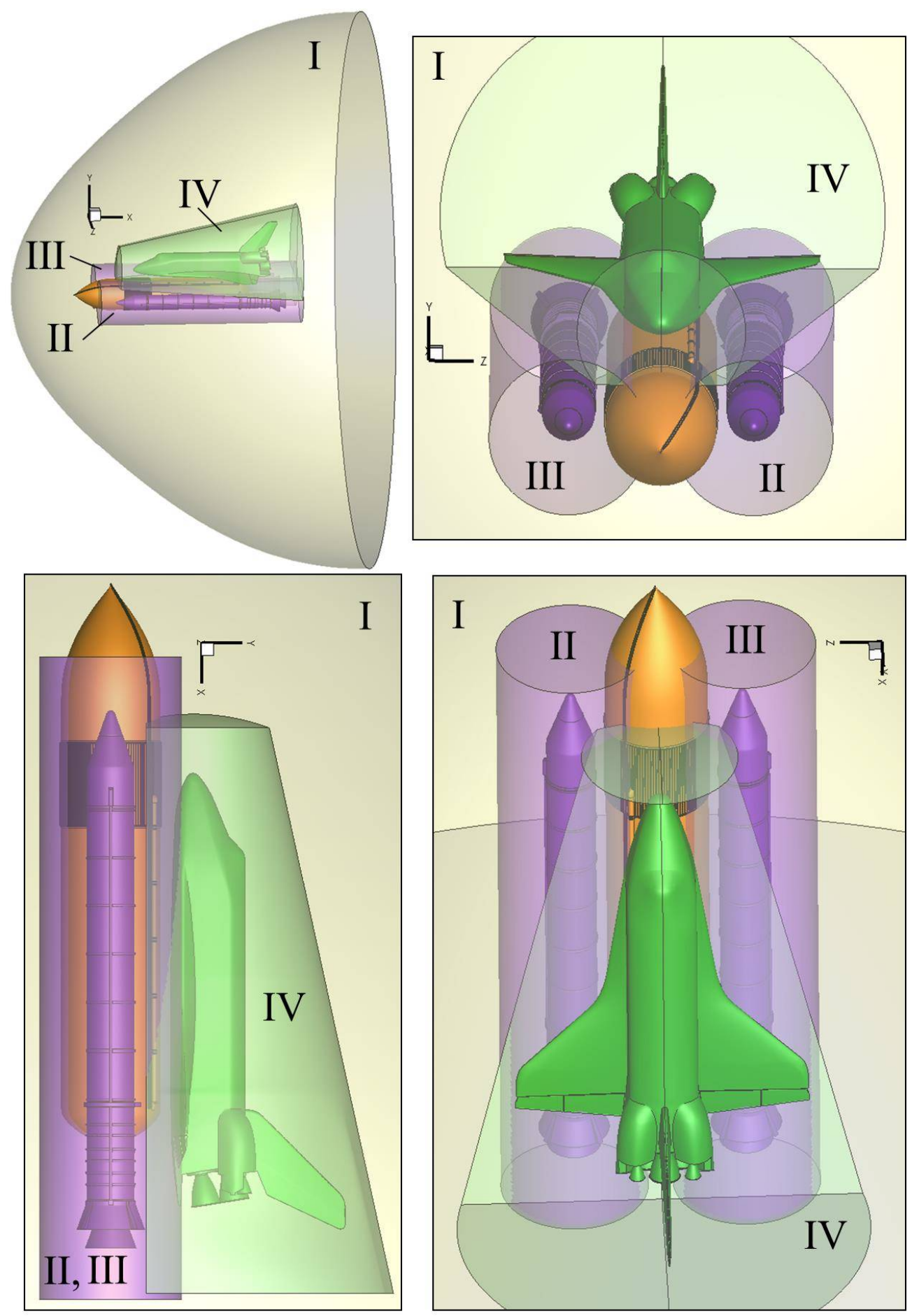

Рис. 10. Перекрывающиеся расчетные сетки, которые независимо построены для каждого компонента транспортной космической системы Space Shuttle. Расположение подобластей соответствует стартовой конфигурации корабля 
Сетка, сгенерированная для конфигурации топливного бака (I), покрывает всю возмущенную область течения. Сеточные модели твердотопливных ускорителей (обозначены на рис.10 римскими цифрами II, III) и орбитальной ступени (IV) полностью погружены в расчетную область I и охватывают зону течения в непосредственной близости от обтекаемых тел.

Дальнейшая реализация технологии построения единой композитной сеточной модели для рассматриваемой четырехкомпонентной системы заключается в исключении части расчетных элементов каждой подобласти из зон их попарного перекрытия. В результате "обрезки" формируются новые границы сеточных областей и узкие зоны взаимного перекрытия заданной ширины (области коммуникации, через которые будет осуществляться информационный обмен между подобластями в процессе численного моделирования). Таким образом, из простого набора сеток может быть получена совокупность взаимосвязанных и взаимодействующих между собой сеточных пространств, составляющих композитную сеточную модель.

Тетраэдральные элементы, подлежащие исключению, объявляются неактивными. Из оставшихся после обрезки активных ячеек выделяется группа коммуникационных элементов, участвующих в интерполяции данных с сетки на сетку [41].

Для поиска неактивных элементов перекрывающихся неструктурированных сеток, которые образуют композитную сеточную модель, разработан полностью автоматизированный алгоритм. Сложность задачи заключается в том, что осуществление поиска "вручную" или даже малейшее вмешательство пользователя в этот процесс не представляется возможным, вследствие неупорядоченной нумерации ячеек и узлов неструктурированных сеток. В настоящем исследовании в качестве параметра автоматического поиска неактивных элементов используется простой критерий расстояния до обтекаемой поверхности.

Рассмотрим первую пару накладывающихся сеток - расчетную область топливного бака (I) и погруженную в нее сетку левого ускорителя (II). Исходя из взаимного положения соответствующих модулей аэрокосмической системы, определим пару величин - значение параметра внешней обрезки расчетной области II ( $L_{\text {cut_out }}^{\mathrm{II}}$ ) и значение параметра внутренней обрезки расчетной области I ( $\left.L_{\text {cut_int }}^{\mathrm{I}}\right)$. В качестве параметра обрезки обеих сеток выбирается расстояние до поверхности левого ускорителя (рис. 11). При этом две выбранные величины $L_{\text {cut_out }}^{\mathrm{II}}$ и $L_{\text {cut_int }}^{\mathrm{I}}$ либо равны между собой (в этом случае область перекрытия усеченных сеточных пространств будет минимальной), либо первое значение незначительно превышает второе $L_{\text {cut_out }}^{\mathrm{II}} \geq L_{\text {cut_int }}^{\mathrm{I}}$. Разница величин задается исходя из среднего размера расчетных ячеек вблизи границ обрезки, и определяет ширину области коммуникации композитной сеточной модели, а также точность интерполяции данных с сетки на сетку.

Процессу выявления неактивных элементов пары накладывающихся расчетных областей топливного бака I и левого ускорителя II предшествует процедура разбиения вершин каждой подобласти на группы активных и неактивных узлов. Последняя демонстрируется на рис. 11 .

Для каждой вершины сетки II определяется ближайшая треугольная ячейка поверхностной сетки твердотопливного ускорителя (исходя из расстояния до центра ячейки). Если расстояние от произвольной вершины до центра ближайшей треугольной площадки на поверхности ускорителя (расстояние до поверхности) превышает $L_{\text {cut_out }}^{\mathrm{II}}$, то вершина переходит в разряд неактивных (см. рис. 11).

Для сеточного пространства топливного бака I процедура поиска неактивных вершин выполняется следующим образом. Для каждой вершины сетки I определяется ближайшая треугольная ячейка поверхностной сетки левого ускорителя. Во-первых, неактивными узлами области І объявляются вершины, попадающие внутрь поверхности ускорителя. Последнее может быть установлено по значению угла между вектором, соединяющим рассматрива- 
емый узел и центр ближайшей треугольной грани поверхностной сетки ускорителя, и внешней нормалью к данной грани. Во-вторых, в число неактивных узлов включаются вершины, удаленные от центра ближайшей треугольной площадки на поверхности ускорителя на расстояние меньшее $L_{\text {cut_int }}^{\mathrm{I}}$ (см. рис. 11).
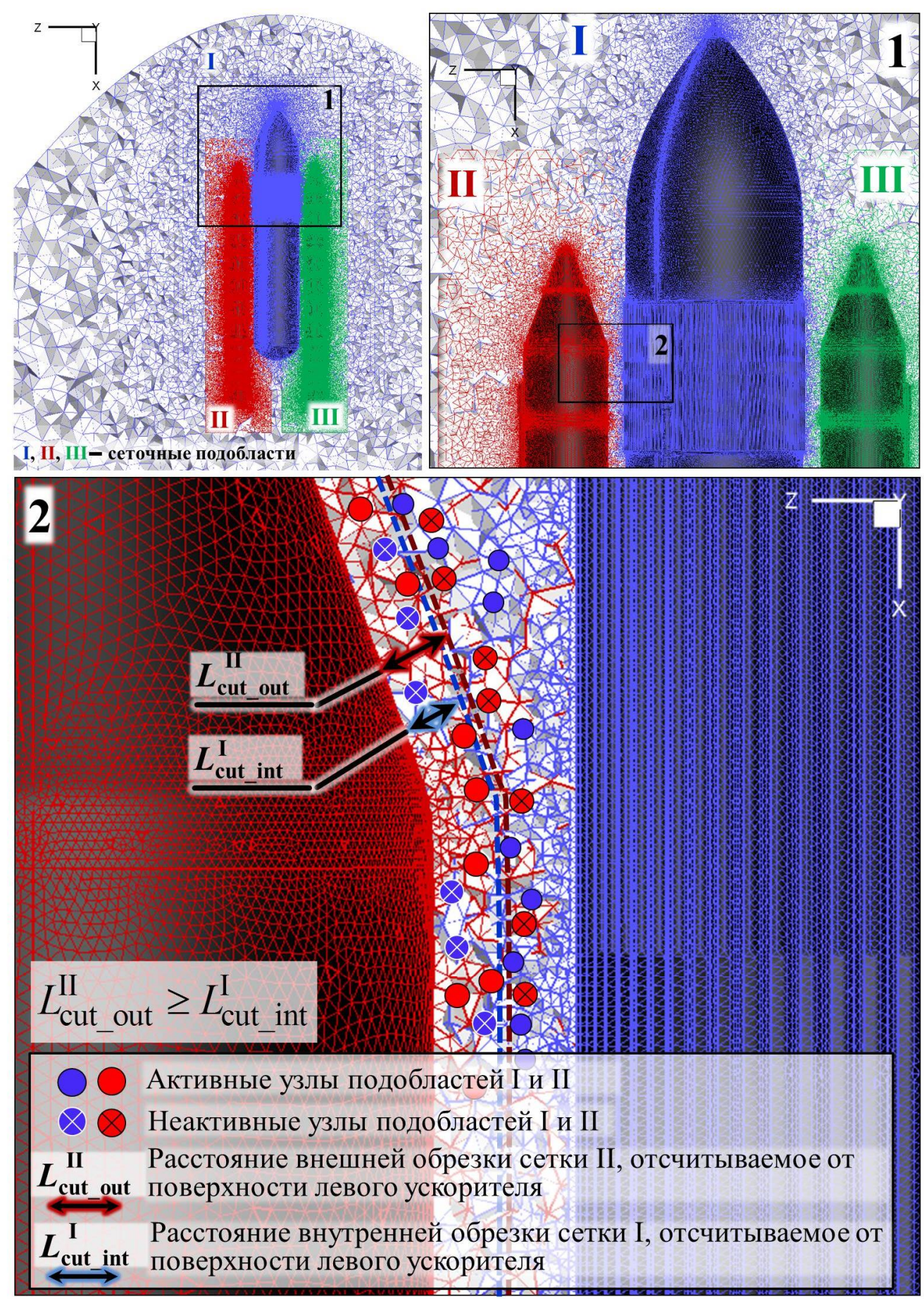

Рис. 11. Определение активных и неактивных узлов для пары пересекающихся сеточных подобластей топливного бака I (синяя сетка) и левого твердотопливного ускорителя II (красная сетка)

На основании полученной классификации узлов расчетные элементы распределяются на две группы: активные ячейки, содержащие хотя бы один активный узел и неактивные элементы, образованные исключительно неактивными узлами. 
На рис. 12 показаны: процедура распределения расчетных элементов пары перекрывающихся двумерных (для большей наглядности) сеток А и В по группам активных и неактивных ячеек (рис. 12, слева), последующее исключение неактивных элементов, а также формирование в результате обрезки новых границ расчетных областей и зоны минимального взаимного перекрытия (рис. 12, справа). Минимизация количества элементов области перекрытия (выделена желтым цветом) упрощает процедуру интерполяции данных с одной сеточной подобласти на другую в ходе вычислений.

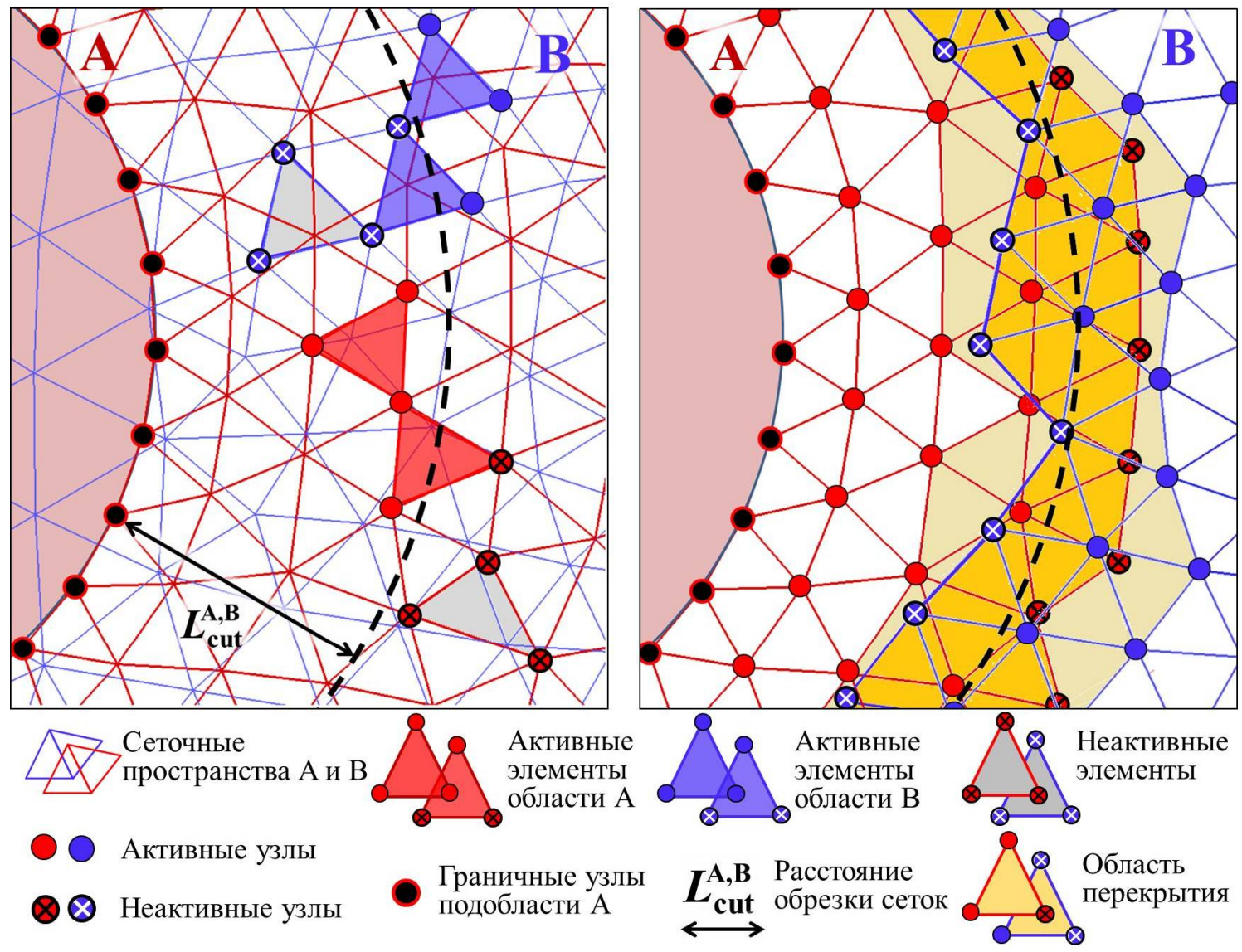

Рис. 12. Процедура определения активных и неактивных элементов (слева) и последующая обрезка сеточных областей А и В с образованием минимальной области перекрытия (справа)

Вернемся к расчетной области подвесного топливного отсека (I) и погруженной в нее расчетной сетке левого твердотопливного ускорителя (II). На рис. 13, слева фиолетовым цветом выделены ячейки исходных (не усеченных) сеточных моделей I и II, попадающие в зону их взаимного перекрытия. Организовать обмен данными между таким большим количеством элементов чрезвычайно сложно, поэтому, следуя изложенной выше методике, необходимо предельно сократить область перекрытия, исключив из рассмотрения часть элементов.

Исходя из определенного выше значения расстояния внешней обрезки сетки левого ускорителя $L_{\text {cut_out }}^{\mathrm{II}}$, удалим из области II неактивные элементы, все вершины которых отдалены от поверхности ускорителя на расстояние, превышающее заданное. Из расчетной области I исключим тетраэдральные ячейки, все узлы которых расположены не далее расстояния внутренней обрезки сетки I ( $L_{\text {cut_int }}^{\mathrm{I}}$ ), а также элементы, попавшие внутрь поверхности ускорителя. На рис. 14 для исходных сеточных пространств I и II представлены распределения тетраэдральных элементов, расположенных в зонах взаимного перекрытия сеток, по отдаленности (в метрах) от поверхности левого твердотопливного ускорителя. 


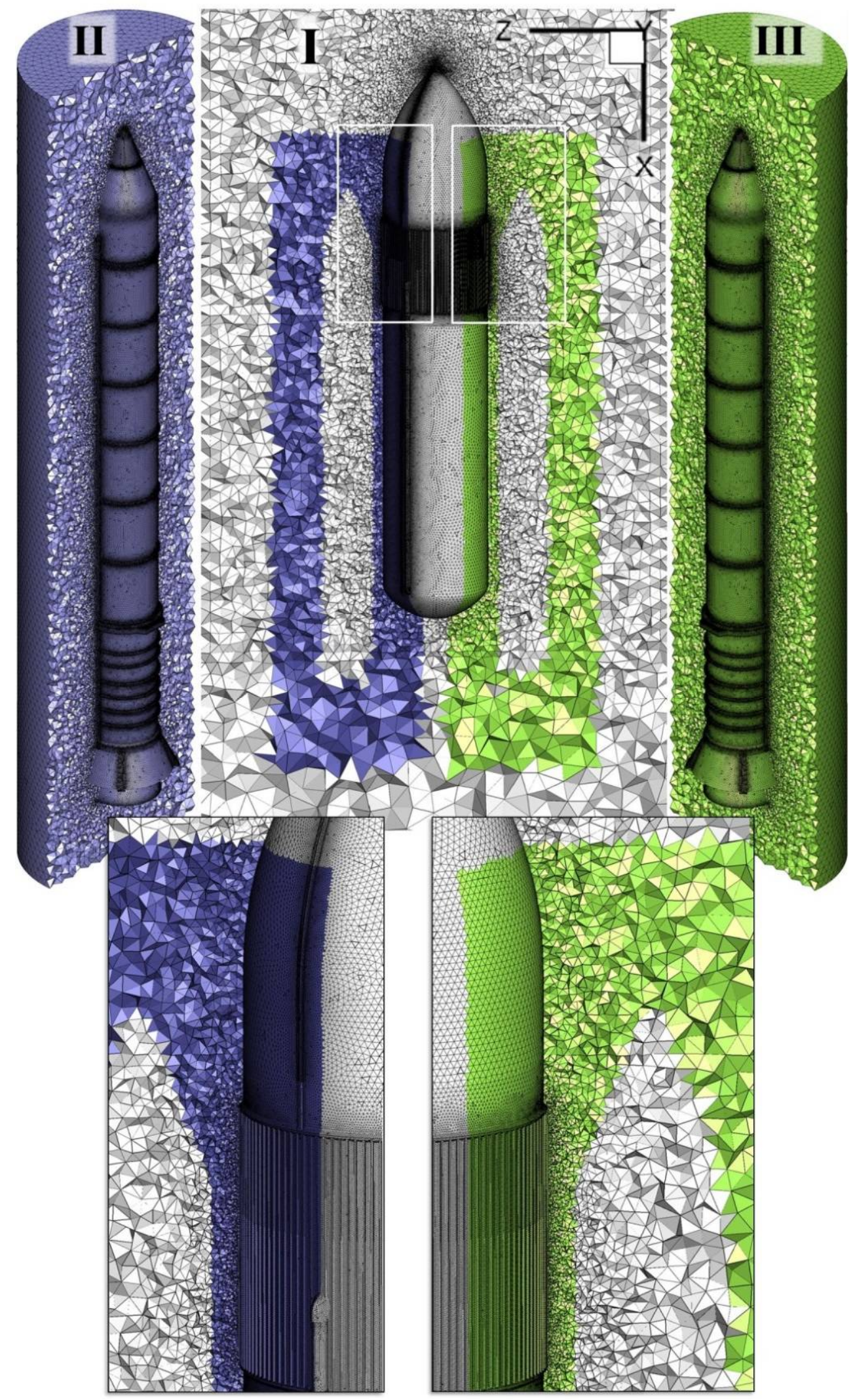

Рис. 13. Расчетные ячейки тетраэдральных сеток топливного бака I и левого ускорителя II, попадающие в область взаимного перекрытия (окрашены фиолетовым цветом). Элементы, оказавшиеся в зоне пересечения сеточных пространств топливного бака I и правого ускорителя III (выделены зеленым цветом) 

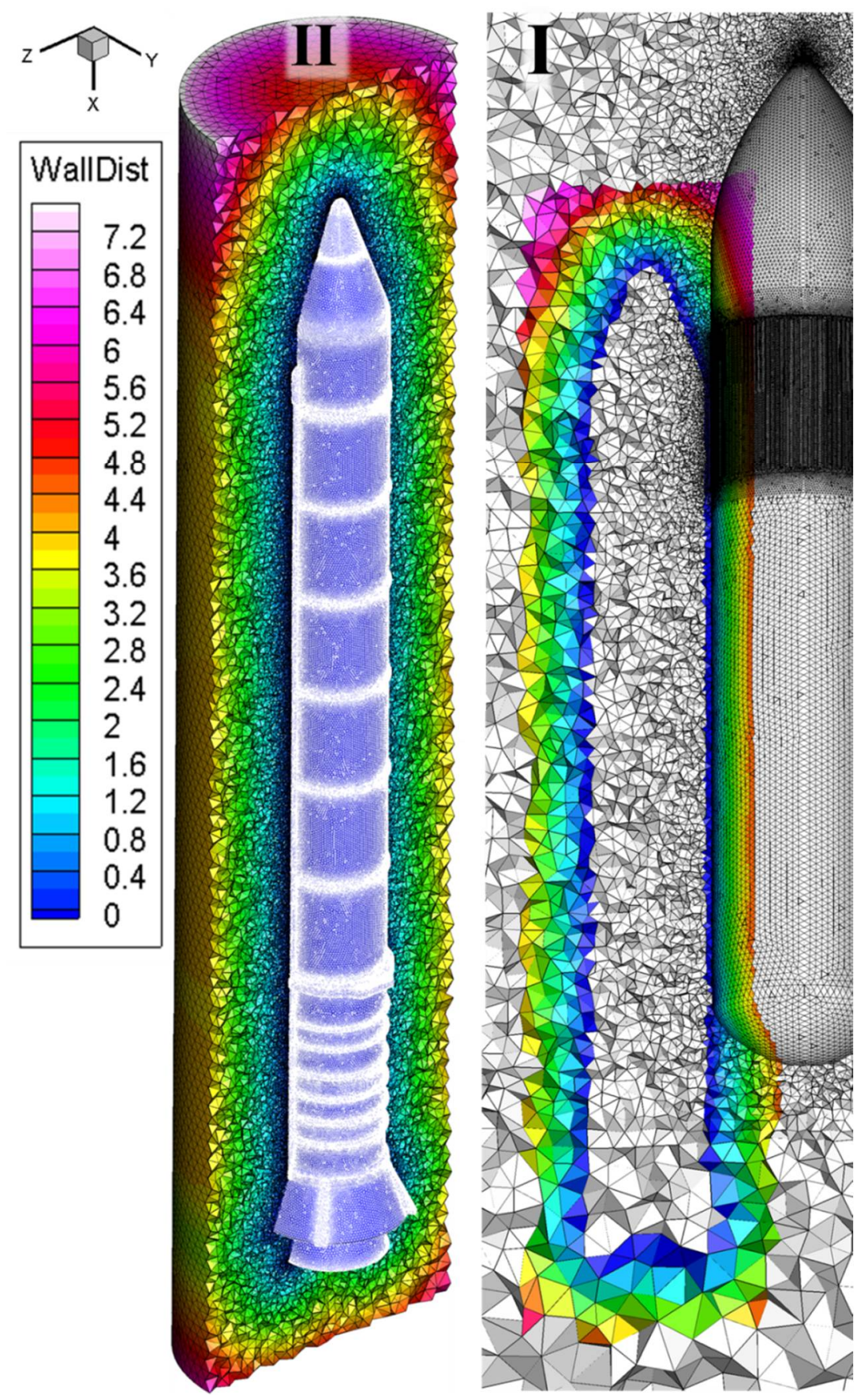

Рис. 14. Распределение элементов подобластей I и II, входящих в область перекрытия, по отдаленности (в метрах) от поверхности твердотопливного ускорителя (расстояние выбрано в качестве критерия для определения неактивных элементов обеих сеточных моделей)

В результате “обрезки” формируются новые внешние границы расчетной области II и внутренние границы расчетной области I (рис. 15, сверху). При объединении усеченных подобластей может быть получена единая композитная сеточная модель с минимальной зоной перекрытия включенных в нее сеток (рис. 15, снизу). При этом ширина области наложения будет достаточной для образования зоны коммуникации, через которую будет осуществляться информационный обмен между подобластями в ходе последующего численного моделирования.

Аналогичным образом проводится взаимная обрезка областей топливного отсека (I) и правого твердотопливного ускорителя (III) с использованием критерия расстояния до поверхности последнего. 


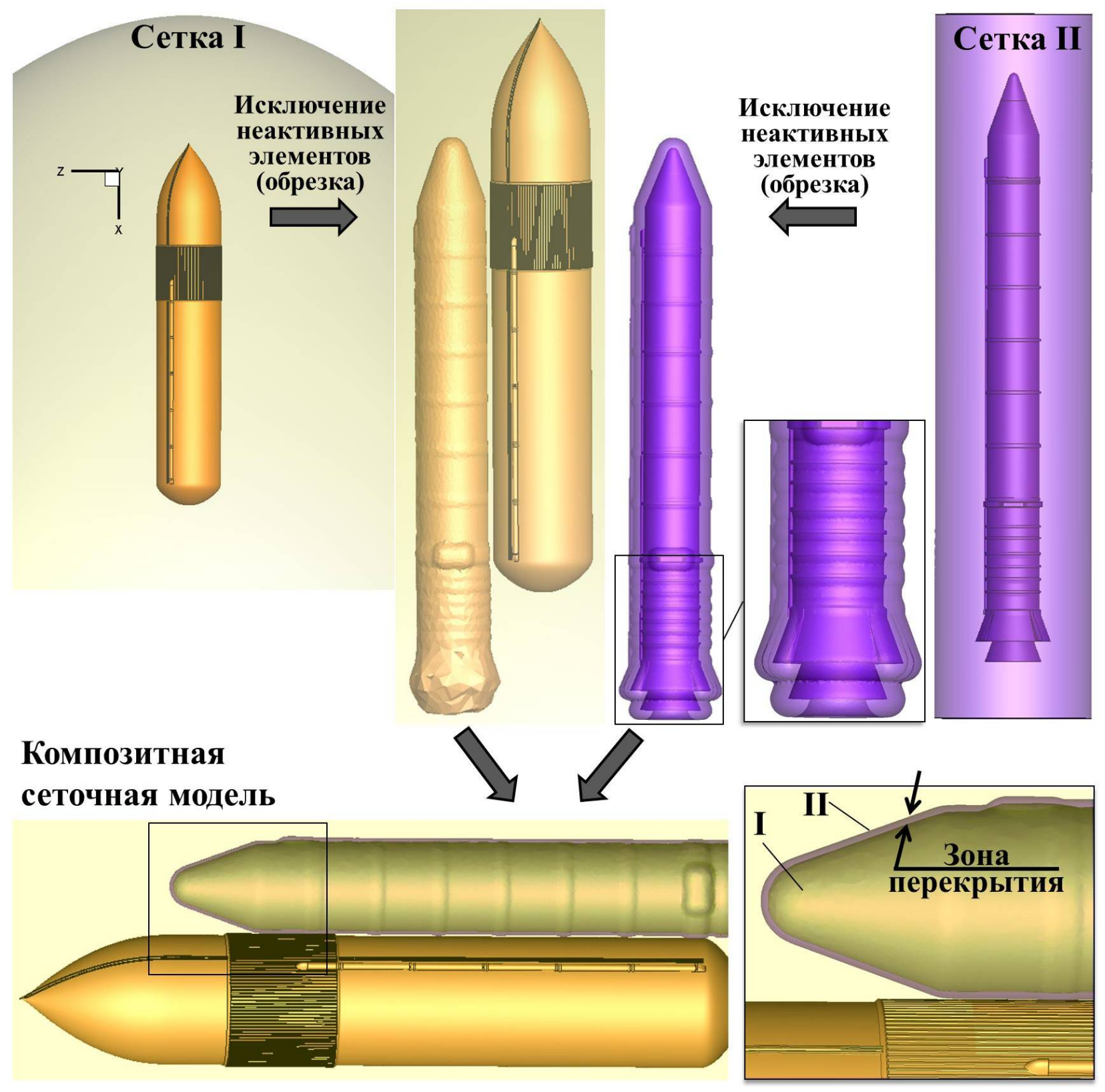

Рис. 15. Исходные и усеченные расчетные области I и II (вверху) и композитная сеточная модель с минимальной зоной перекрытия (внизу), построенная для компоновки топливный бак - левый ускоритель с использованием предложенной технологии

Рисунки 16 и 17 поясняют ход описанной выше процедуры исключения неактивных тетраэдральных элементов для сеток топливного бака I и орбитальной ступени IV из зоны их перекрытия. На рис. 16 расчетные ячейки обеих сеточных областей, попадающие в область наложения сеток, выделены желтым цветом. В данном случае в качестве критерия внутренней и внешней обрезки сеточных пространств используется отдаленность расчетных узлов от поверхности орбитальной ступени. На рис. 17, сверху представлены распределения элементов подобластей I и IV по выбранному критерию. Рисунок 17, снизу иллюстрирует результат усечения неструктурированной сетки IV, независимо сгенерированной около поверхности орбитального ракетоплана. 


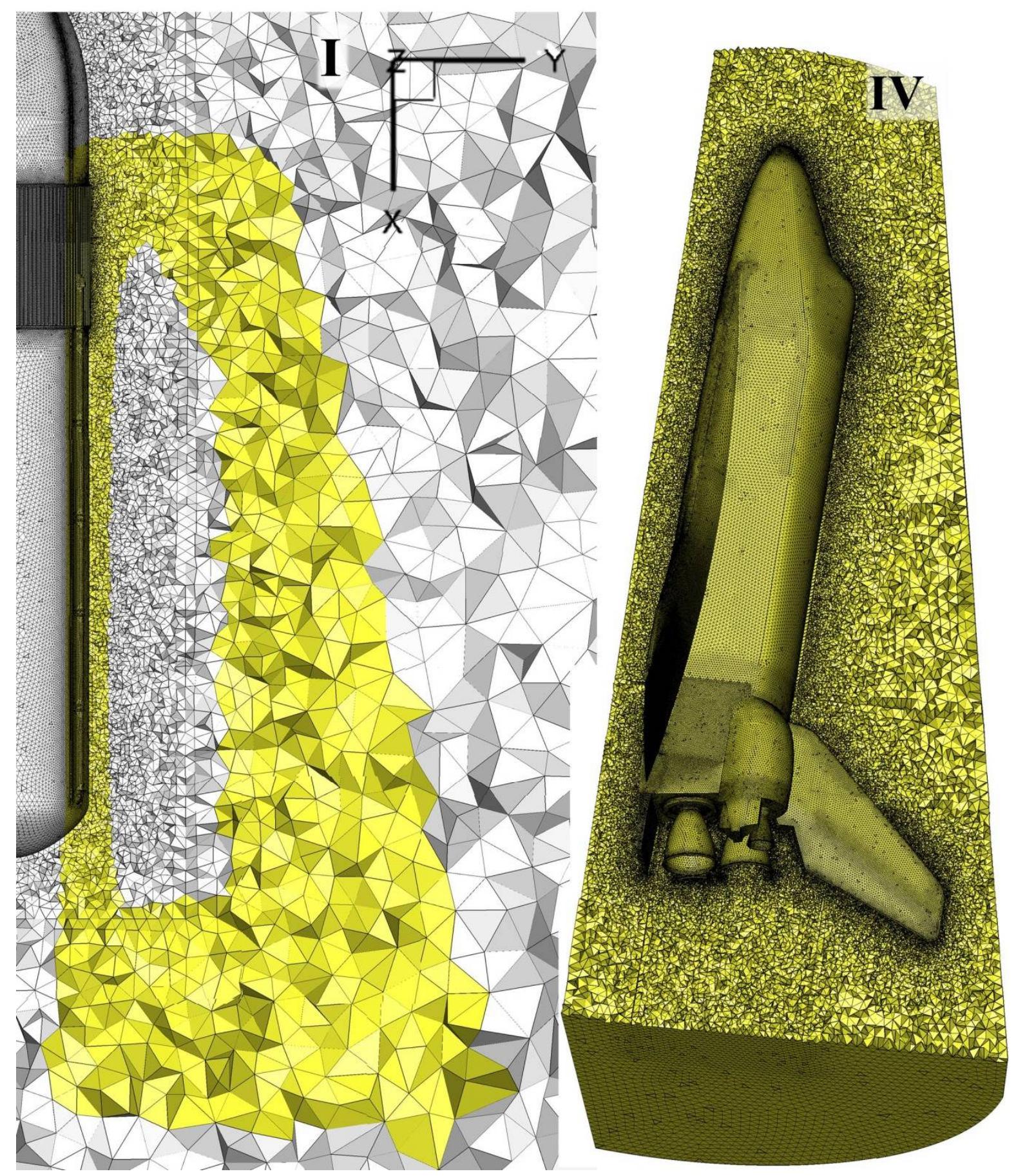

Рис. 16. Расчетные ячейки тетраэдральных сеток топливного бака I и орбитальной ступени $\mathrm{IV}$, попадающие в область взаимного перекрытия (окрашены в желтый цвет) 

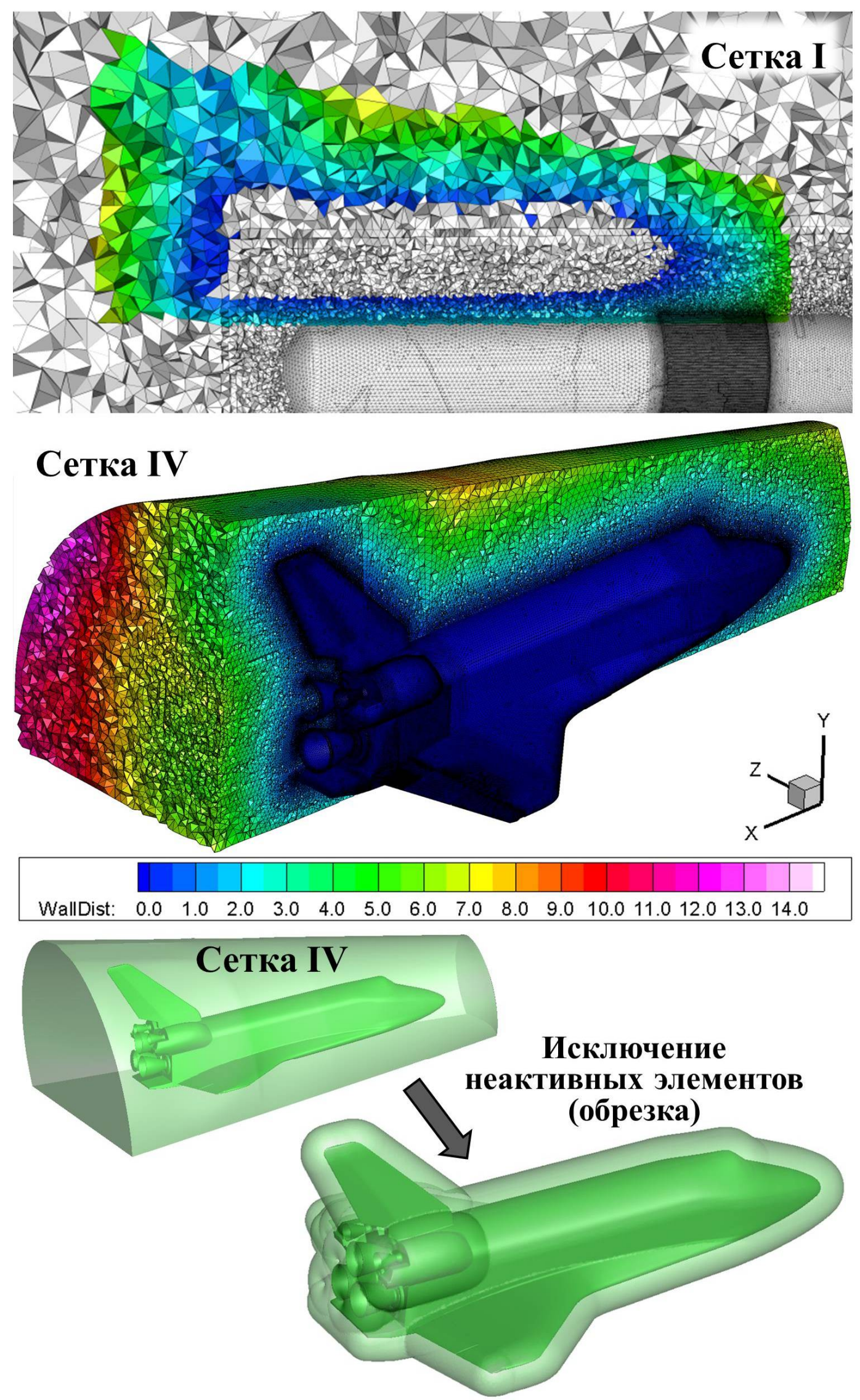

Рис. 17. Распределение расчетных ячеек сеточных моделей топливного бака (I) и орбитальной ступени (IV) в зависимости от расстояния (в метрах) до поверхности орбитера и последующая обрезка области IV по соответствующему критерию 
Композитная сеточная модель космического корабля Space Shuttle, которая объединяет в себе усеченные сеточные подпространства, независимо построенные для всех модулей системы в стартовой конфигурации, показана на рис. 18. Расчетные сетки топливного бака (I), орбитальной ступени (IV), левого и правого ускорителей (II, III), включенные в интегрированную модель, имеют минимальные области попарного перекрытия.

Использование предложенной композиционной модели позволяет избежать многократного перестроения расчетных сеток при изменении числа или взаимного положения компонентов системы. Минимизация количества расчетных элементов в зонах взаимного пересечения сеток максимально сокращает протяженность области коммуникации (области информационного обмена между сетками) и упрощает процедуру интерполяции данных с одной сеточной подобласти на другую в ходе последующих вычислений.

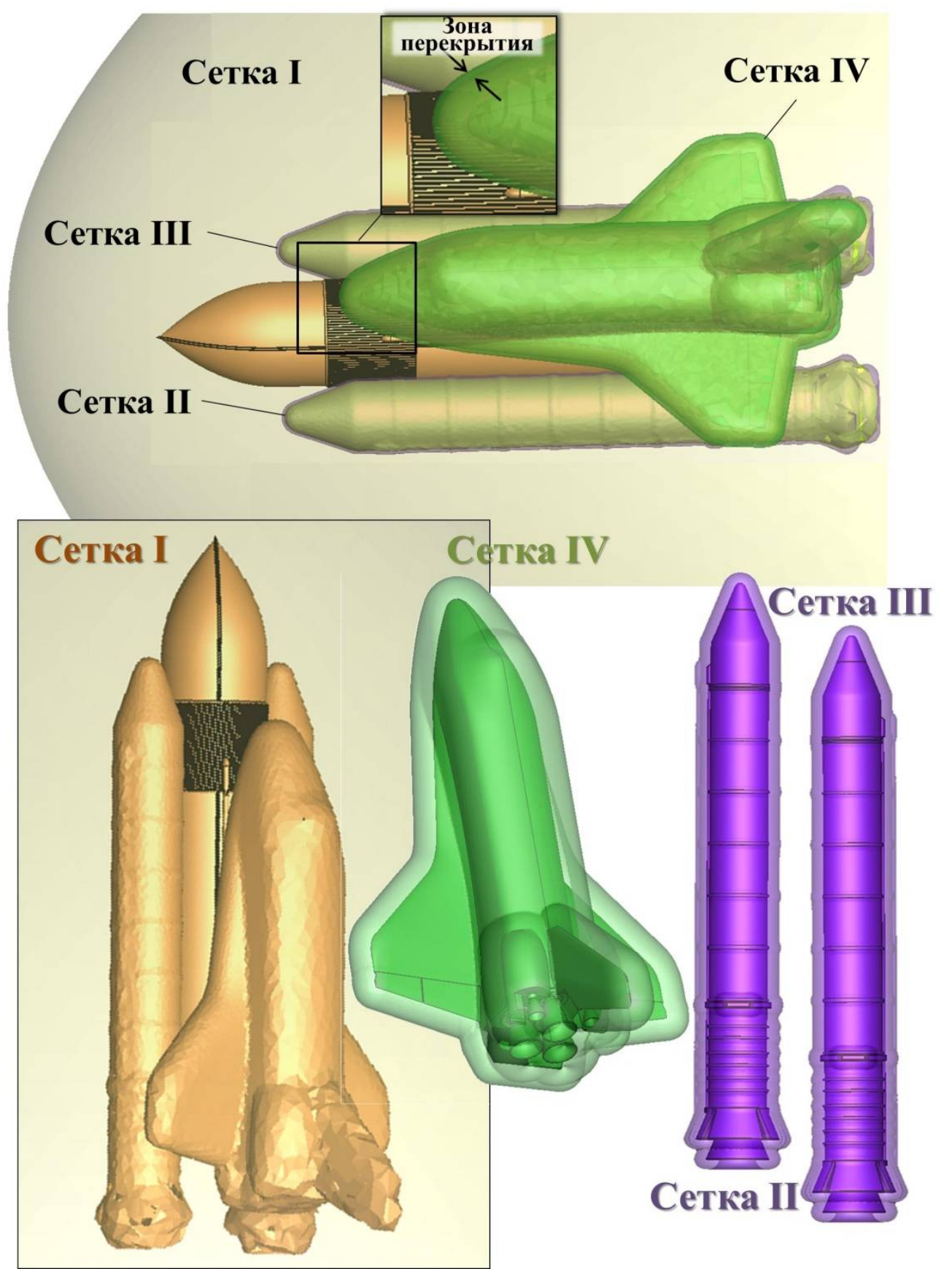

Рис. 18. Композитная неструктурированная сеточная модель для численного моделирования обтекания многоступенчатой космической системы Space Shuttle в полной компоновке 


\section{5. Реализация алгоритмов интегрирования уравнений газовой динамики на композитной неструктурированной сетке}

Вычислительная модель аэротермодинамики системы произвольно расположенных тел сложной конфигурации - модулей космического корабля Space Shuttle во взлетной конфигурации, построена на основе технологий решения полной системы уравнений механики сплошной среды (уравнений Навье-Стокса), разработанных в ИПМех РАН [15-23].

Для решения трехмерной задачи течения совершенной вязкой сжимаемой среды система нестационарных уравнений Навье - Стокса может быть записана в векторном виде

$$
\frac{\partial \mathbf{w}}{\partial t}+\frac{\partial \mathbf{F}^{\mathbf{x}}(\mathbf{w})}{\partial x}+\frac{\partial \mathbf{F}^{\mathbf{y}}(\mathbf{w})}{\partial y}+\frac{\partial \mathbf{F}^{\mathbf{z}}(\mathbf{w})}{\partial z}=\frac{\partial \mathbf{G}^{\mathbf{x}}(\mathbf{w})}{\partial x}+\frac{\partial \mathbf{G}^{\mathbf{y}}(\mathbf{w})}{\partial y}+\frac{\partial \mathbf{G}^{\mathbf{z}}(\mathbf{w})}{\partial z},
$$

где $\mathbf{w}=(\rho, \rho u, \rho v, \rho w, \rho E)^{T}-$ столбец консервативных переменных;

$\mathbf{F}^{\mathbf{x}}=\left(\rho u, \rho u^{2}+p, \rho u v, \rho u w, \rho u E+p u\right)^{T}, \mathbf{F}^{\mathbf{y}}=\left(\rho v, \rho u v, \rho v^{2}+p, \rho v w, \rho v E+p v\right)^{T}$,

$\mathbf{F}^{\mathbf{z}}=\left(\rho w, \rho u w, \rho v w, \rho w^{2}+p, \rho w E+p w\right)^{T}-$ проекции вектора конвективного потока;

$\mathbf{G}^{\mathbf{x}}=\left(0, \tau_{x x}, \tau_{y x}, \tau_{z x}, u \tau_{x x}+v \tau_{y x}+u \tau_{z x}-q_{x}\right)^{T}, \mathbf{G}^{\mathbf{y}}=\left(0, \tau_{x y}, \tau_{y y}, \tau_{z y}, u \tau_{x y}+v \tau_{y y}+w \tau_{z y}-q_{y}\right)^{T}$,

$\mathbf{G}^{\mathbf{Z}}=\left(0, \tau_{x z}, \tau_{y z}, \tau_{z z}, u \tau_{x z}+v \tau_{y z}+w \tau_{z z}-q_{z}\right)^{T}-$ проекции вектора вязкого потока;

$\rho$ - плотность; $p$ - давление; $u, v, w$ - компоненты вектора скорости; $E$ - удельная полная энергия газа; $\tau_{\alpha \beta}-$ компоненты тензора вязких напряжений $(\alpha=x, y, z ; \beta=x, y, z) ; q_{x}, q_{y}, q_{z}$ - компоненты вектора теплового потока. Система уравнений (1) замыкается с использованием уравнения состояния совершенного газа.

Исходный газодинамический решатель, ориентированный на тетраэдральные расчетные сетки, реализует метод расщепления по физическим процессам $[42,43]$ исходной нестационарной системы уравнений Навье-Стокса (1). Стационарное решение задачи, если оно существует, получается в результате установления.

Расчет каждого временного шага разбивается на несколько известных этапов [42, 43]. На первом этапе определяются промежуточные значения параметров потока без учета эффектов переноса. На втором этапе вычисляются эффекты переноса, учитывающие обмен между элементами - рассчитываются потоки массы через границы расчетных ячеек. На третьем этапе определяются в новый момент времени окончательные значения газодинамических параметров потока на основе законов сохранения массы, импульса и энергии для каждого элемента и всей системы в целом.

Для аппроксимации уравнений на каждом этапе применяются элементы метода конечных объемов. В рамках данного подхода определяются усредненные значения производных по контрольному объему (тетраэдральному элементу).

$$
\begin{gathered}
\left\langle\frac{\partial f}{\partial \alpha}\right\rangle_{V_{i}}=\frac{1}{V_{i}} \int_{V_{i}} \frac{\partial f}{\partial \alpha} \mathrm{d} V=\frac{1}{V_{i}} \int_{S_{i}} f \mathbf{i} \cdot \mathrm{d} \mathbf{S} \approx \frac{1}{V_{i}} \sum_{j=1}^{4} S_{i}^{j} n_{i, j}^{\alpha} f_{i}^{j}, \\
f=\left(u, v, w, p, q_{\alpha}, \tau_{\alpha \beta}\right), i=1 \ldots N, j=1 \ldots 4, \alpha=x, y, z ; \beta=x, y, z
\end{gathered}
$$

Здесь $V_{i}$ - объем $i$-го тетраэдра; $S_{i}^{j}$ - площадь поверхности $j$-й грани $i$-го элемента; $n_{i, j}^{\alpha}-x$, $y$ или $z$-я составляющая единичной нормали $j$-й грани $i$-го элемента. Реализация метода расщепления по физическим процессам подразумевает решение большого количества однотипных подзадач по вычислению потоков через грани контрольных объемов. 
Для повышения порядка точности численной схемы с сохранением ее устойчивости, в рамках метода расщепления по физическим процессам, применялся метод аппроксимации потоков AUSM (Advection Upstream Splitting Method) [44], основанный на расщеплении вектора потоков искомых параметров на границах расчетных ячеек.

Реализация модели трехмерного течения сжимаемого газа на композитной сетке не потребовала значительной модификации исходных кодов. Однако для адаптации алгоритмов интегрирования полной системы уравнений Навье-Стокса были учтены следующие особенности композиционной сеточной модели. Во-первых, вместо исходных границ подобластей (или в дополнение к ним) рассматривались новые границы, сформированные в процессе исключения неактивных элементов (обрезки). Во-вторых, была реализована дополнительная процедура интерполяции данных с сетки на сетку в областях взаимного перекрытия.

Рисунок 19 иллюстрирует процесс интерполяции для двух пересекающихся сеточных подобластей А и В, образующих минимальную, но достаточную для взаимного информационного обмена, зону перекрытия (выделена желтым цветом). На рисунке для большей наглядности рассмотрен двумерный случай. При переходе на пространственные сетки принцип интерполяции не меняется. Сплошные заполненные красным или синим цветом кружки, как было определено на рис. 12, обозначают активные узлы соответствующих неструктурированных сеток А и В, кружки с крестиками обозначают неактивные вершины (последние являются граничными узлами соответствующих подобластей). Для каждого узла, расположенного на обновленной границе подобласти определяется “донорная ячейка" - элемент второй подобласти, в которую попадает данный узел. В примере, показанном на рис. 19 (крупно в центре), одна из граничных вершин сетки В попадает внутрь “донорного элемента" $M N K$, принадлежащего второй подобласти А. Совокупность всех граничных узлов обеих областей и соответствующие “донорные ячейки” образуют зону коммуникации сеточных моделей А и В (элементы коммуникационной зоны обеих сеток окрашены в желтый цвет).

Переменная $Q$ в произвольной точке донорного элемента восстанавливается по трем известным значениям параметра в центре текущей ячейки и двух ближайших центрах смежных элементов с применением кусочно-линейной реконструкции. В настоящем исследовании используемый интерполяционный шаблон соответствует случаю, когда переменные определены в центрах расчетных ячеек.

Для линейного распределения параметра внутри ячейки вычисляется градиент с использованием значений в центрах текущей и соседних ячеек $Q_{i}, Q_{j}, Q_{k}$ (рис. 19).

$$
\begin{gathered}
\nabla Q=\left(Q_{x}, Q_{y}\right)^{T}, \\
Q_{x}=\frac{\left|\begin{array}{ll}
Q_{j}-Q_{i} & y_{j}-y_{i} \\
Q_{k}-Q_{i} & y_{k}-y_{i}
\end{array}\right|}{\left|\begin{array}{ll}
x_{j}-x_{i} & y_{j}-y_{i} \\
x_{k}-x_{i} & y_{k}-y_{i}
\end{array}\right|}, Q_{y}=\frac{\left|\begin{array}{cc}
x_{j}-x_{i} & Q_{j}-Q_{i} \\
x_{k}-x_{i} & Q_{k}-Q_{i}
\end{array}\right|}{\left|\begin{array}{ll}
x_{j}-x_{i} & y_{j}-y_{i} \\
x_{k}-x_{i} & y_{k}-y_{i}
\end{array}\right|}
\end{gathered}
$$

Общая формула реконструкции значения параметра $Q$ в произвольной точке $i$-й ячейки может быть записана следующим образом:

$$
Q\left(x_{o}, y_{o}\right)=Q_{i}+\nabla Q \cdot\left(\mathbf{r}_{o}-\mathbf{r}_{i}\right)
$$

Для трехмерного случая процесс интерполяции выполняется аналогичным образом, например, с использованием четырех значений газодинамических параметров, определенных в центре рассматриваемого донорного элемента и трех ближайших центрах смежных с ним тетраэдральных ячеек. 


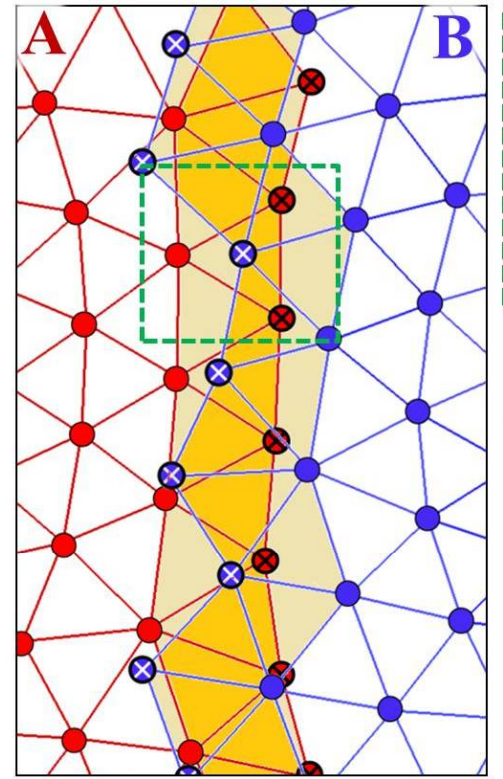

$\mathbf{r}_{\boldsymbol{i}}$ Радиус-вектор центра $i$-й ячейки

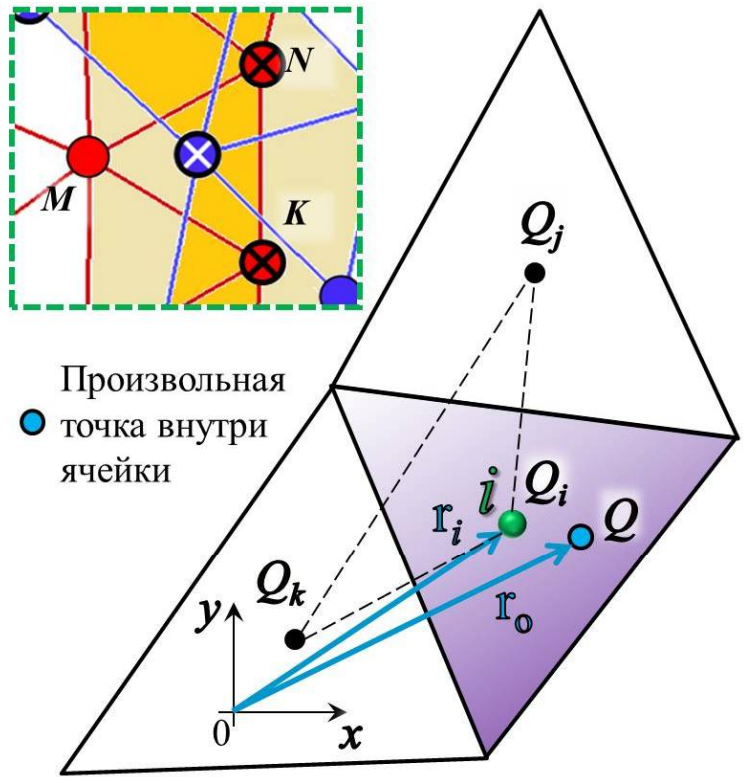

$\mathbf{r}_{\mathbf{o}}$ Радиус-вектор произвольной точки внутри ячейки
$Q_{i}\left(x_{i}, y_{i}\right)$,
$Q_{j}\left(x_{j}, y_{j}\right)$,
$Q_{k}\left(x_{k}, y_{k}\right)$
Значения параметра $Q$ в центрах текущей и смежных ячеек $i, j, k$ с соответствующими координатами
Значение параметра, восстановленное в произвольной точке $i$-й ячейки

Рис. 19. Восстановление значения параметра $Q$ в произвольной точке элемента $i$ по трем известным значениям соответствующего параметра в центрах текущей и соседних ячеек

\section{6. Результаты численного моделирования}

Пример применения изложенной методики композитных сеток в рамках модифицированной модели аэротермодинамики летательных аппаратов произвольной конфигурации, разработанной в Лаборатории радиационной газовой динамики ИПМех РАН [15-23], приведен на рис. 20. Для демонстрации возможностей метода выполнено моделирование обтекания космической транспортной системы Space Shuttle во взлетной конфигурации. Моделируется фаза подъема на этапе, предшествующем отделению ускорителей [45]. На рисунке показана сложная структура трехмерного течения, формирующаяся вблизи космического корабля Space Shuttle в полной компоновке. Результаты представлены в виде распределений давления по поверхностям отдельных модулей и поля давлений в центральном сечении. Параметры набегающего потока соответствуют одной из точек номинальной траектории космического корабля [45] - высоте $H=40$ км, числу Маха $\mathrm{M}_{\infty}=4$, углу атаки $\alpha=4^{0}$.

\section{7. Заключение}

Рассмотрена концепция композитных неструктурированных сеток для численного моделирования сложных многоступенчатых аэрокосмических систем с изменяемым взаимным положением и числом элементов. В работе продемонстрировано, что технология, основанная на представлении сеточной модели в виде набора независимо построенных сеток, успешно используется не только в рамках блочно-структурированного подхода.

Показано, что неструктурированные композитные сетки могут применяться для моделирования сложных трехмерных течений, благодаря возможности дискретизировать расчетную область произвольной формы и относительно легко адаптироваться в зонах больших градиентов. 


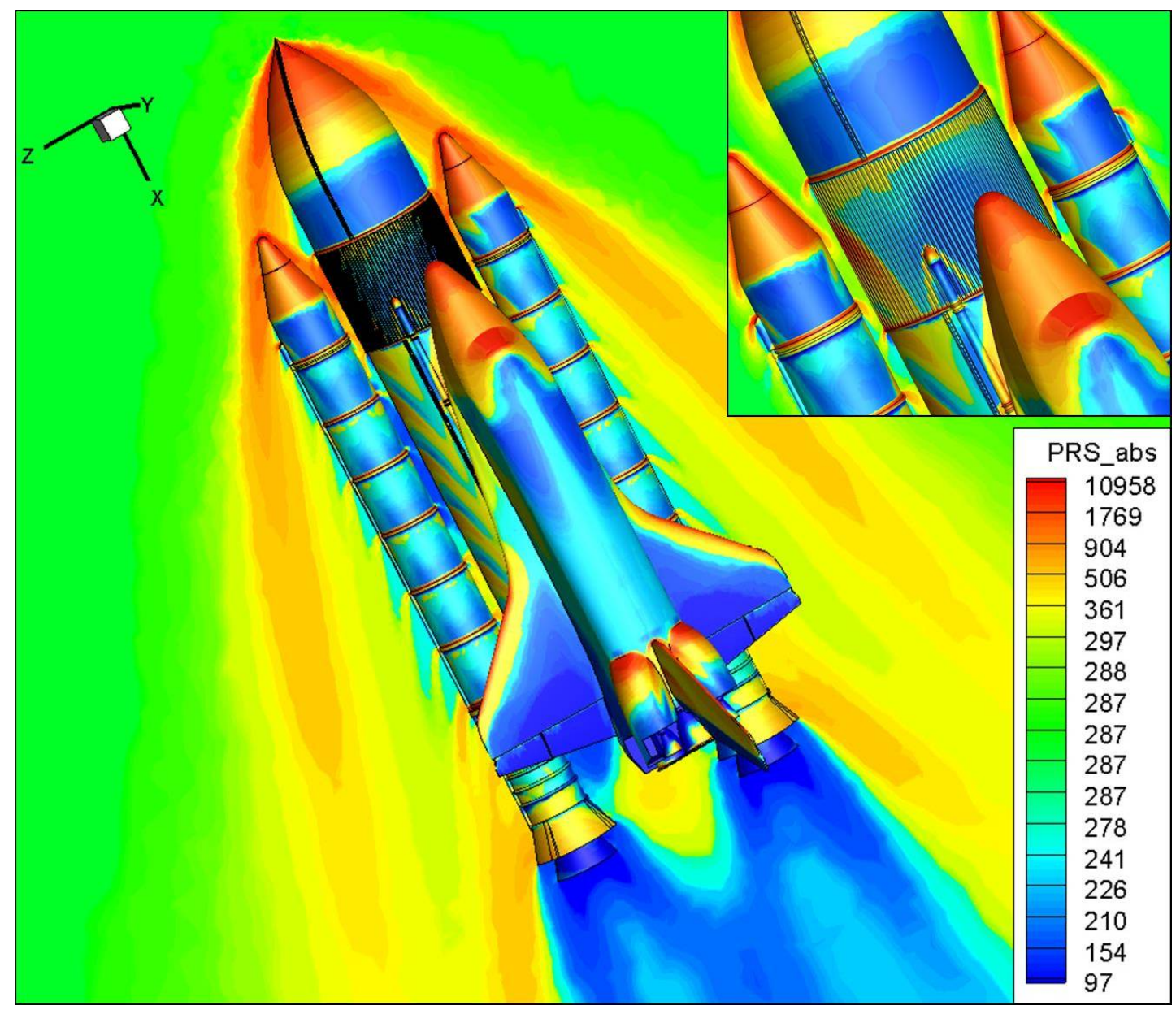

Рис. 20. Возмущенное пространственное поле течения около сложной конфигурации многокомпонентной космической транспортной системы Space Shuttle во взлетной конфигурации. Распределение давления вблизи космического корабля и на его поверхности при скорости набегающего потока $\mathrm{M}_{\infty}=4$ и угле атаки $\alpha=4^{0}$ на высоте 40 км

Кроме этого, в отличие от альтернативного блочно-структурированного подхода, число сеток не возрастает с увеличением сложности геометрии расчетной области, что позволяет сократить вычислительные затраты при интерполяции данных между сетками. Использование минимального числа неструктурированных сеточных подобластей, независимо сгенерированных для отдельных элементов компоновки, позволяет избежать перестроения сеток при изменении взаимного положения совместно обтекаемых тел, что позволяет значительно экономить временные ресурсы. Минимизация количества расчетных элементов в зонах взаимного пересечения сеток максимально сокращает протяженность области коммуникации и упрощает процедуру интерполяции данных с одной сеточной подобласти на другую в ходе вычислений. При относительно небольших дополнительных вычислительных затратах на интерполяцию данных, подход дает значительный выигрыш в производительности.

Рассмотренная методика построена на основе модификации разрабатываемых в ИПМех РАН моделей аэротермодинамики тел произвольной конфигурации и их адаптации к использованию на композитных перекрывающихся неструктурированных сетках.

Возможности предложенной технологии продемонстрированы на примере расчета пространственного поля течения около космического транспортного корабля Space Shuttle в полной компоновке, состоящей из орбитального самолета, подвесного топливного отсека и двух твердотопливных ускорителей. Создан виртуальный прототип сложной системы, который объединяет в себе набор объемных сеточных моделей, независимо сгенерированных на основе максимально реалистичных цифровых поверхностей. 
Метод дает возможность рассматривать большое число конфигураций многокомпонентной системы в кратчайшие сроки, обеспечивая достаточную стабильность и приемлемую точность численных результатов, что особенно важно при моделировании аэродинамики разделяющихся ступеней.

Автор выражает благодарность своему научному руководителю, академику РАН, проф. С.Т. Суржикову за идеи, положенные в основу работы.

Работа выполнена в Лаборатории радиационной газовой динамики ИПМех РАН в рамках Программы фундаментальных исследований Российской академии наук, при поддержке гранта РФФИ 16-01-00379.

\section{Литература}

1. NSTS 1988 News Reference Manual: Space Transportation System. https://science.ksc.nasa.gov/shuttle/technology/sts-newsref/

2. Jenkins D.R. Space Shuttle: The History of Developing the National Space Transportation System. $1996,320 \mathrm{p}$.

3. История разработки многоразовой транспортно-космической системы (МТКС) “Спейс Шаттл”. http://www.buran.ru/htm/shuttle.htm

4. Многоразовый орбитальный корабль “Буран”. http://www.buran.ru/htm/mtkkmain.htm

5. отогалерея NASA. http://spaceflight.nasa.gov/gallery/images/shuttle/

6. Железнякова А.Л. Унифицированный подход к созданию сложных виртуальных поверхностей и расчетных сеток для комплексного имитационного 3D моделирования современных изделий аэрокосмической техники // Физико-химическая кинетика в газовой динамике. 2016. Том 17, вып. 2.24 c. http://chemphys.edu.ru/issues/2016-17-2/articles/634/

7. Rogers D.F. An Introduction to NURBS with Historical Perspective. Morgan Kaufman Publishers, San Fransisco, 2001. 324 p.

8. $\quad$ Piegl L.A., Tiller W. The NURBS Book. Springer, 1997. 646 p.

9. Lee K. Principles of CAD/CAM/CAE Systems. Addison-Wesley, California, 1999. 582 p.

10. SolidWorks. http://www.solidworks.com/

11. Железнякова А.Л., Суржиков С.Т. Построение пространственных неструктурированных сеток на NURBS-поверхностях сложных изделий авиационной и ракетно-космической техники методом молекулярной динамики //Физико-химическая кинетика в газовой динамике. 2014. Т.15, вып. 1. http://chemphys.edu.ru/issues/2014-15-1/articles/108/

12. Zheleznyakova A.L. Molecular dynamics-based triangulation algorithm of free-form parametric surfaces for computer-aided engineering // Computer Physics Communication. 2015. http://dx.doi.org/10.1016/j.cpc.2014.12.018.

13. Zheleznyakova A.L., Surzhikov S.T. Molecular dynamic-based unstructured grid generation method for aerodynamic application // Computer Physics Communication, Vol.184, 2013. Pp.2711-2727.

14. Железнякова А.Л. Молекулярно-динамический метод построения неструктурированных сеток в сложных пространственных областях и на криволинейных поверхностях //Физико-химическая кинетика в газовой динамике. 2012. Т.13, вып. 4. http://chemphys.edu.ru/issues/2012-134/articles/368/

15. Железнякова А.Л. Моделирование аэротермодинамических характеристик виртуального прототипа перспективного сверхзвукового авиалайнера на крейсерском режиме полета//Физикохимическая кинетика в газовой динамике. 2017. T.18, вып. 1. http://chemphys.edu.ru/issues/2017$18-1 /$ articles/672/

16. Железнякова А.Л., Суржиков С.Т. Расчет гиперзвукового обтекания тел сложной формы на неструктурированных тетраэдральных сетках с использованием схемы AUSM // TBT, 2014, т. 52, № 2, c. 283-293.

17. Железнякова А.Л., Суржиков С.Т. Применение метода расщепления по физическим процессам для расчета гиперзвукового обтекания пространственной модели летательного аппарата сложной формы // ТВТ, 2013, том 51, № 6, с. 897-911.

18. Железнякова А.Л., Суржиков С.Т. На пути к созданию модели виртуального ГЛА. І. - М.: ИПМех РАН, 2013. - 160 с. 
19. Железнякова А.Л., Суржиков С.Т. Численное моделирование поля течения при входе в атмосферу земли спускаемого аппарата с аэродинамическим качеством // Вестник МГТУ им. Н.Э. Баумана. - Сер. "Машиностроение". 2009. №2. С.3-25.

20. Железнякова А.Л., Суржиков С.Т. Численное моделирование гиперзвукового обтекания модели летательного аппарата X-43 // Препр. ИПМех им. А.Ю. Ишлинского РАН. № 950. 2010. 82 с.

21. Железнякова А.Л., Кузенов В.В., Петрусев А.С., Суржиков С.Т. Численный анализ конвективного нагрева двух моделей спускаемых космических аппаратов // Вестник МГТУ им. Н.Э. Баумана. - Сер. "Машиностроение". 2009. №3. С.3-15.

22. Железнякова А.Л., Суржиков С.Т. Численное моделирование гиперзвукового обтекания модели летательного аппарата Х-43 // Вестник МГТУ им. Н.Э. Баумана. - Сер. "Машиностроение". 2010. №1. C.3-19.

23. Железнякова А.Л., Кузенов В.В., Петрусев А.С., Суржиков С.Т. Расчет аэротермодинамики двух типов моделей спускаемых космических аппаратов. // Физико-химическая кинетика в газовой динамике. 2010. Том 9. http://chemphys.edu.ru/media/published/025.pdf

24. Iliff K.W., Shafer M.F. Space Shuttle Hypersonic Aerodynamic and Aerothermodynamic Flight Research and the Comparison to Ground Test Results / NASA Technical Memorandum 4499, 1993.

25. Young J.C., Underwood J.M., Hillje E.R. The Aerodynamic Challenges of the Design and Development of the Space Shuttle Orbiter. NASA Report, 1985.

26. Bornemann W.E., Surber T.E. Aerodynamic Design of the Space Shuttle Orbiter. AGARD CPP-247, paper 11, Sept. 1978. 24 p.

27. Muylaert J., Walpot L., Rostand P., Rapuc M., Brauckmann G., Paulson J., Trockmorton D., Weilmuenster K. Extrapolation from wind tunnel to flight: Shuttle orbiter aerodynamics. NASA Technical Report. 1998. 16 p.

28. Arrington J.P., Jones J.J. Shuttle Performance: Lessons Learned, Part 1. NASA-CP-2283-PT-1. 1983. $760 \mathrm{p}$.

29. Arrington J.P., Jones J.J. Shuttle Performance: Lessons Learned, Part 2. NASA-CP-2283-PT-2. 1983. $647 \mathrm{p}$.

30. Aerodynamic Design Data Book. Orbital Vehicle STS-1. Rockwell International. 1980. 1640 p.

31. Space Shuttle: Solid Rocket Boosters / NASA. http://www.nasa.gov/returntoflight/system/ system_SRB.html

32. Moore D.R., W.J. Phelps. Reusable Solid Rocket Motor - Accomplishments, Lessons, and a Culture of Success // AIAA Space 2011 Conference and Exposition, Long Beach, CA. 23 p.

33. Space Shuttle: The External Tank. / NASA. https://www.nasa.gov/returntoflight/system/ system_ET.html

34. Железнякова А.Л. Компьютерное моделирование спуска орбитальной ступени космической системы Space Shuttle в плотных слоях атмосферы Земли // Физико-химическая кинетика в газовой динамике. 2017. Т.18, вып. 2. 39 c. http://chemphys.edu.ru/issues/2017-18-2/articles/716/

35. Encyclopedia Astronautica. Index: O. OME. http://www.astronautix.com/o/ome.html

36. Fundamentals of Space Systems / V.L. Pisacane, R.C. Moore (Editors), Oxford University Press, New York. 1994. 772 p.

37. Harland D.M. The Story of the Space Shuttle. Springer-Verlag, Praxis Publishing Ltd. 2004. 444 p.

38. Owen J.W. Shuttle Propulsion Overview - the Design Challenges / NASA-CR-2012-2485. 2012. 15p.

39. Liseikin V.D. Grid Generation Methods. - Berlin: Springer, 1999.

40. Thompson J.F., Soni B. K., Weatherill N. P. Handbook of Grid Generation. CRC Press, 1998.

41. Nakahashi K., Togashi F. Overset unstructured grid method for flow simulation of complex and multiple body problems / ICAS 2000 CONGRESS. ICAS 0263. 10 p.

42. Белоцерковский О.М., Давыдов Ю.М. Метод крупных частиц в газовой динамике. - М.: Наука, 1982. - 391 с.

43. Марчук Г.И. Методы расщепления. - М: Наука, 1988. - 263 с.

44. Liou M. S., Steffen C. A New Flux Splitting Scheme - J. Comput. Phys., Vol. 107, 23-39, 1993.

45. Martin F.W., Slotnick J.P. Flow Computations for the Space Shuttle in Ascent Mode Using Thin-Layer Navier-Stokes Equations // Applied Computational Aerodynamics, P.A. Henne, ed., AIAA, 1990, pp. 863-886. 